\title{
Article \\ Age-Related Alterations in EEG Network Connectivity in Healthy Aging
}

\author{
Hamad Javaid ${ }^{1}$, Ekkasit Kumarnsit ${ }^{2,3}$ and Surapong Chatpun ${ }^{1,3,4, *(\mathbb{C})}$
}

1 Department of Biomedical Sciences and Biomedical Engineering, Faculty of Medicine, Prince of Songkla University, Hat Yai, Songkhla 90110, Thailand; qazihummad@gmail.com

2 Physiology Program, Division of Health and Applied Science, Faculty of Science, Prince of Songkla University, Hat Yai, Songkhla 90112, Thailand; ekkasit.k@psu.ac.th

3 Biosignal Research Centre for Health, Prince of Songkla University, Hat Yai, Songkhla 90112, Thailand

4 Institute of Biomedical Engineering, Faculty of Medicine, Prince of Songkla University, Hat Yai, Songkhla 90110, Thailand

* Correspondence: surapong.c@psu.ac.th

Citation: Javaid, H.; Kumarnsit, E.; Chatpun, S. Age-Related Alterations in EEG Network Connectivity in Healthy Aging. Brain Sci. 2022, 12, 218. https://doi.org/10.3390/ brainsci12020218

Academic Editor: Ricardo Bajo

Received: 4 January 2022

Accepted: 1 February 2022

Published: 5 February 2022

Publisher's Note: MDPI stays neutral with regard to jurisdictional claims in published maps and institutional affiliations.

Copyright: (c) 2022 by the authors. Licensee MDPI, Basel, Switzerland. This article is an open access article distributed under the terms and conditions of the Creative Commons Attribution (CC BY) license (https:// creativecommons.org/licenses/by/ $4.0 /)$.

\begin{abstract}
Emerging studies have reported that functional brain networks change with increasing age. Graph theory is applied to understand the age-related differences in brain behavior and function, and functional connectivity between the regions is examined using electroencephalography (EEG). The effect of normal aging on functional networks and inter-regional synchronization during the working memory (WM) state is not well known. In this study, we applied graph theory to investigate the effect of aging on network topology in a resting state and during performing a visual WM task to classify aging EEG signals. We recorded EEGs from 20 healthy middle-aged and 20 healthy elderly subjects with their eyes open, eyes closed, and during a visual WM task. EEG signals were used to construct the functional network; nodes are represented by EEG electrodes; and edges denote the functional connectivity. Graph theory matrices including global efficiency, local efficiency, clustering coefficient, characteristic path length, node strength, node betweenness centrality, and assortativity were calculated to analyze the networks. We applied the three classifiers of K-nearest neighbor $(\mathrm{KNN})$, a support vector machine (SVM), and random forest (RF) to classify both groups. The analyses showed the significantly reduced network topology features in the elderly group. Local efficiency, global efficiency, and clustering coefficient were significantly lower in the elderly group with the eyes-open, eyes-closed, and visual WM task states. KNN achieved its highest accuracy of $98.89 \%$ during the visual WM task and depicted better classification performance than other classifiers. Our analysis of functional network connectivity and topological characteristics can be used as an appropriate technique to explore normal age-related changes in the human brain.
\end{abstract}

Keywords: EEG; graph theory; aging; working memory; classification

\section{Introduction}

The human brain is a complex structural and functional network organ in the human body. Graph theory was introduced to study the complex network organization of the brain. The structural and functional systems of the brain have the characteristics of a complex network, such as network modularity, highly connected hubs, and small world topology [1,2]. Electroencephalography (EEG) studies have shown reduced connectivity in adults to be influenced by aging in a resting state through a difficult mathematical calculation [3]. In other EEG studies, network connectivity was reduced in an elderly group compared to a younger group $[4,5]$. In order to study the network properties of brain networks, several network matrices were used to investigate brain aging, such as characteristic path length, node strength, edge and node betweenness centrality, clustering coefficient, global efficiency, and local efficiency [6]. The study of brain networks in healthy aging, especially age-related changes in memory, plays a vital role in understanding the 
deficits created by Alzheimer's disease (AD). Graph theory is applied to model the human brain as a complex network represented by nodes or vertices (i.e., brain regions) connected by edges (i.e., functional connections) [1,7].

The changes in brain networks occur dynamically in response to various contexts or external stimuli, even in a resting state [8-10]. In eyes-open and eyes-closed conditions, an age-related decrease in reactivity was found in alpha and beta bands [11,12]. In a resting state, EEG study path length and clustering coefficient were decreased in most of the frequency bands in elderly individuals, which was associated with a more random network topology [5]. Moreover, the variation in brain network dynamics has been linked with human learning [13], cognitive function [14], healthy aging [15,16], and mental disorders [17].

The slowing in cognitive processing speed is associated with age and it reflects cognitive decline in elderly individuals [18]. Working memory (WM) involves the capacity to temporarily hold information and manipulate it for short period of time. WM can be divided into three stages: the initial encoding of information, the maintenance of WM items, and the retrieval of WM items [19]. Prominent age-related differences were seen in older adults in WM tasks compared to a short-term memory task that required only the maintenance and storage of information [20]. Several studies have utilized the n-back test to investigate age-related alterations in the brain, revealing the underlying mechanism by applying functional magnetic resonance imaging (fMRI) $[21,22]$ and EEG $[23,24]$. The brain oscillatory responses vary during the phases of working memory while engaging in a WM task. Studies using the Sternberg item recognition task found distinct patterns for activation in the encoding, storage, and retrieval of WM, which were found to be sensitive to WM load levels [25-28].

Machine learning (ML) approaches have been widely used in bio-signal analysis and disease classification. ML techniques have been used in emotion recognition [29] and the prediction of diseases including dementia [30], stroke [31], and AD [32]. Furthermore, ML techniques have been used on EEG signals to understand their complex electrophysiological activities and characterize the dynamic features of a complex brain network. Several studies have utilized traditional machine learning models such as the K-nearest neighbor (KNN), decision tree (DT), random forest (RF), Naïve Bayes, and regression models to investigate neurological disorders $[33,34]$. In a recent study, support vector machines (SVM), KNN, and Naïve Bayes were used to predict the AD [32] and SVM, correctly classifying 83\% of the subjects using network features. To classify healthy aging EEG signals using network features, SVM achieved an accuracy above 80\% [6]. A low-density device with seven electrodes was designed for an automated EEG-based AD detection system, and the SVM obtained 91.1\% accuracy [35]. A portable EEG device was used to quantify the mental workload during the driving and the binary machine learning models achieved high accuracy (98.2\% to $99.6 \%)$ between resting state and driving state [36]. Furthermore, a low-cost EEG system was developed to predict ischemic stroke events, and the SVM model obtained $92 \%$ accuracy [37]. In the automatic detection of epileptic EEG, ML framework based on RF combined with a grid search optimization technique achieved an accuracy of $96.7 \%$ [38].

Network analysis has been used to investigate the network dynamics of neurological diseases such as AD and mild cognitive impairment [39-41]. Resting state connectivity and network topology are increasingly being studied to understand the effect of aging on specific brain regions connected in a resting state. Previous studies have shown that reduced small world configuration and increased path length reduce clustering in the resting state network in healthy aging EEG signals $[6,18]$. It has been recommended that network indices of graph theory can be used to investigate the age-related characteristic of functional networks of the brain [42]. Moreover, graph theory has been applied to investigate age-related alterations during an n-back test using a clustering coefficient, a small world coefficient, and characteristic path length [43]. In order to explore how a resting state network configuration involving regions is different from a WM state 
configuration, our current work focuses on age-related differences in networks in a resting state and under visual working memory conditions. However, to the best of our knowledge, network analysis has not been applied in a visual working memory task to investigate agerelated changes in middle-aged and elderly populations and understand the mechanism of cognitive aging.

We hypothesized that simple tasks, both resting and visual WM tasks, and features of graph theory are useful tools to differentiate between age-related changes in EEG signals of the human brain. Therefore, this study aimed to investigate the age-related differences in EEG networks in middle-aged and elderly individuals in a resting state and during a visual WM task. We recorded EEG from 20 healthy middle-aged subjects and 20 healthy elderly subjects in eyes-open and eyes-closed states, as well as during a visual WM task. In our work, several network features based on graph theory, including global efficiency, local efficiency, clustering coefficient, characteristic path length, node strength, and assortativity, are extracted from both groups. We additionally applied SVM, KNN, and RF algorithms to classify healthy aging EEG signals using functional network characteristics. The results of this study can pave the way on early cognitive aging detection using EEG signals with simple tasks and features of graph theory.

We organized our study into five sections, including "Introduction". The methodology and experimental details are presented in the "Materials and Methods", the "Results" demonstrate our findings, and the "Discussion" compares our results with those from other studies. In the last section, "Conclusions", we conclude the findings of our study.

\section{Materials and Methods}

\subsection{Participants}

The study protocol was approved by the human research ethical committee of our institution (HSC-HREC-61-006-02-1). All the participants signed informed consent letters, and the experiment was explained to them. We acquired EEGs from two age groups, a middle-aged group (age range, 41 to 60 years; mean age $=50.50 \pm 5.77$ years) and an elderly group (age range, 61 to 84 years; mean age $=71.03 \pm 5.45$ years). Each group consisted of 20 healthy participants who had no previous history of any psychological or neurological disorder.

\subsection{Experimental Task}

We acquired an EEG from all participants in an eyes-open state for 5 min, an eyesclosed state for $5 \mathrm{~min}$, and during a visual WM task. Figure 1 illustrates the experimental procedure for EEG acquisition. This WM task was suggested to study alterations in a healthy human EEG [44]. Before the experiment, the task was explained to all participants. All necessary instructions were shown on a monitor screen. Participants were asked to focus on a screen displaying a "+" symbol for $30 \mathrm{~s}$. Three major steps were involved in this WM task. In the first step, 25 images were shown on the screen for $30 \mathrm{~s}$; in the second step, those images were removed from the screen and the participants had to memorize the images in $30 \mathrm{~s}$ while their eyes remained closed. In last step, the participants were instructed to open their eyes and recall the images in $30 \mathrm{~s}$. The total number of correct answers was recorded as the WM performance score.

\subsection{EEG Recording and Preprocessing}

We used an Ultracortex Mark IV headset (OpenBCI, New York, NY, USA). Eight electrodes (FP1, FP2, C3, C4, P7, P8, O1, and O2) and reference electrodes on both ear lobes were applied according to the 10-20 international system for electrode placement. OpenBCI has been used previously to predict the amplitude modulation of steady-state, visually evoked potentials signals with a single electrode [45]. OpenBCI has been used to develop EEG-based applications, including a device for disabled people [46], and to assess P3, N2, and FRN components for performance monitoring [47]. In a comparison of dry and wet electrode EEG systems, the dry electrode device was found to be more 
robust to $50 \mathrm{~Hz}$ line noise, less sensitive to electromagnetic interference, and useful for self-application and home usage [48]. The $250 \mathrm{~Hz}$ sampling frequency was used to acquire the EEG. Pre-processing was carried out to remove the noise and artefacts from the data. We used EEGLAB toolbox for preprocessing and FastICA algorithm was used to remove the artifacts from the electrooculography (EOG) and electromyography (EMG) artifacts out of the EEG signals [49]. A $50 \mathrm{~Hz}$ infinite impulse response notch filter was used to clean the AC powerline noise. Impedance was kept below $5 \mathrm{k} \Omega$. EEG data were filtered into a 0.5-45 Hz band using the band pass finite impulse response filter (2nd order Butterworth. For analysis, MATLAB R2019b (MathWorks Inc., Natick, MA, USA) was used.

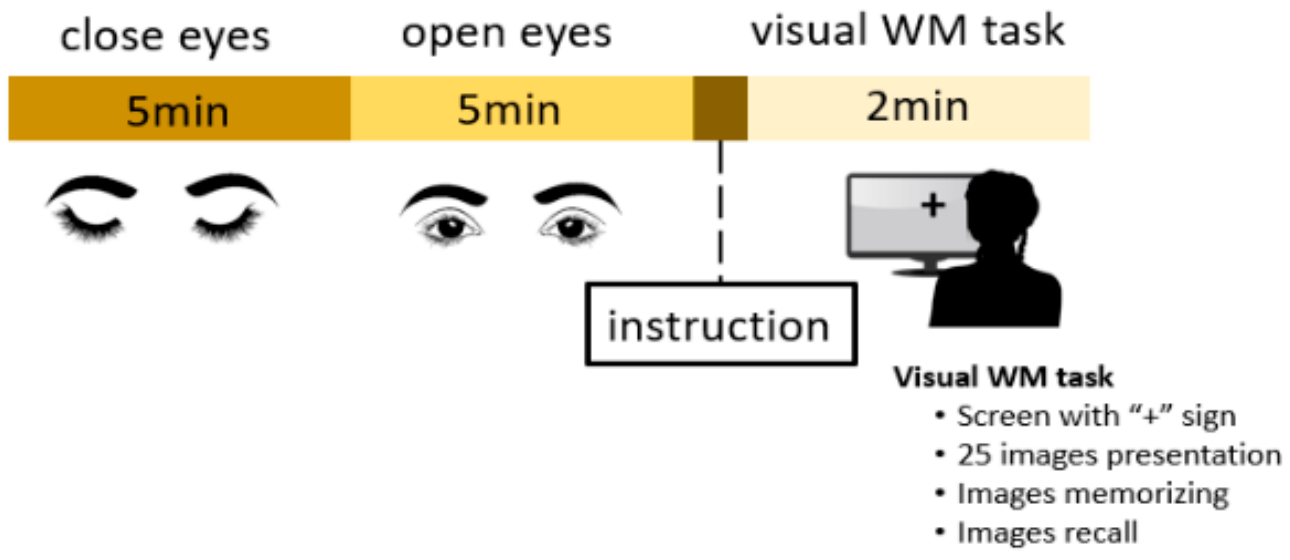

Figure 1. Illustration of experimental protocol.

\subsection{Network Construction}

To construct the functional network, the first step is to obtain the connectivity matrices representing inter-relations between brain regions. In order to obtain connectivity matrices, different methods were proposed [50]. In this study, we used Pearson's correlation, which has been frequently used in functional network construction. The correlation coefficient between two electrodes, $x$ and $y$, can be calculated as [32]:

$$
r_{x, y}=\frac{\operatorname{cov}(x, y)}{\sqrt{\operatorname{var}(x) \operatorname{var}(y)}}
$$

where $\operatorname{cov}(\mathrm{x}, \mathrm{y})$ is the covariance between node $\mathrm{x}$ and node $\mathrm{y}$, and $\operatorname{var}(\mathrm{x})$ and $\operatorname{var}(\mathrm{y})$ are the variances of node $x$ and node $y$, respectively.

To reduce the noise level, binary networks are often constructed from weighted connectivity matrices. For this purpose, thresholding of the correlation matrices is utilized. If the correlation weight between two nodes is greater than a certain threshold, this represents a relation between the nodes. A certain threshold value is used to threshold the weighted correlation matrices [51]. In the sparsity thresholding method, all extracted networks have the same density; thus, the comparison is unbiased in relation to density.

In this current work, seven features of graph theory were calculated for both age groups. Local efficiency, global efficiency, characteristic path length, clustering coefficient, node betweenness centrality, node strength, and assortativity were calculated for the analysis.

These network measures correspond to the communicability of brain regions, the connectivity structure, segregation phenomena, and synchronization in a weighted graph. These network features can be computed as shown in the literature [7,32]. Local efficiency of node $\mathrm{i}\left(\mathrm{LocE}_{\mathrm{i}}\right)$ is computed as shown in Equation (2).

$$
\operatorname{LocE}_{i}=\frac{1}{d_{i}\left(d_{i}-1\right)} \sum_{j=g_{i}} \frac{1}{L_{i, j}}
$$


where $d_{i}$ represents the degree of the node (number of nodes connected to node $i$ ), $L_{i, j}$ shows shortest path length between nodes $i$ and $j$, and $g_{i}$ is a graph of neighbors of node $i$ excluding node $i$. The local efficiency of the network is calculated by taking the average of all nodes, as shown in Equation (3).

$$
\operatorname{Loc} E=\frac{1}{N} \operatorname{LocE}_{\mathrm{i}}
$$

Here, $\mathrm{N}$ is the total number of nodes. The human brain processes information in a specific manner while each brain region processes a specific kind of information; this is called the segregation of information. The local efficiency and clustering coefficient are the network measures used for this purpose. Clustering coefficient (CC) quantifies the intensity of the neighbors of the connected nodes in a network [6].

$$
C C=\frac{1}{N} \sum_{h} \frac{\sum_{i, j} a_{i j} a_{i h} a_{j h}}{d_{h}\left(d_{h}-1\right)}
$$

where $d_{h}$ is the degree of the node and $a_{i j}$ is a member of the connectivity matrix.

Global efficiency (GlobE) measures communication efficiency in a network, and it is inversely proportion to its average shortest path length. Global efficiency can be calculated as in $[7,32]$.

$$
\text { GlobE }=\frac{1}{\mathrm{~N}(\mathrm{~N}-1)} \sum_{\mathrm{i}, \mathrm{j}} \frac{1}{\mathrm{~L}_{\mathrm{i}, \mathrm{j}}}
$$

while analyzing the weighted networks, the degree of the network was extended to the sum of the weights in the network [52]. Node strength (S) can be computed as show below [7].

$$
S(i)=\sum_{j}^{n} M_{i j}
$$

$M$ represents the weighted connectivity matrix; $M_{i j}$ is greater than 0 if node $j$ is connected to node i.

The significance of nodes and edges is measured by calculating the edges and node betweenness of the centrality features. Node betweenness centrality (NB) is calculated using the Equation (7) [7,32].

$$
\mathrm{NB}_{(\mathrm{u})}=\sum_{\mathrm{u}=\mathrm{v}=\mathrm{w}} \frac{\mathrm{P}_{\mathrm{v}, \mathrm{w}}(\mathrm{u})}{\mathrm{P}_{\mathrm{v}, \mathrm{w}}}
$$

where $P_{v, w}$ is the number of shortest paths between nodes $v$ and $w$, and $P_{v, w}(u)$ denotes the number of shortest paths between nodes $v$ and $w$ passing through node $u$.

The characteristic path length (CP) measures the mean path length in the network, and it is computed using the following Equation [6,7].

$$
C P=\frac{1}{n} \sum_{i \in N} L_{i}=\frac{1}{n(n-1)} \sum_{i \in N} \sum_{j \in N, i \neq j} d_{i j}
$$

where $L_{i}$ is the average path length between node $i$ and all other nodes, and $d_{i j}$ is the distance between node $\mathrm{i}$ and node $\mathrm{j}$ in the network.

A network may experience random failure in its components. Resiliency against failures is vital for the proper functioning of the network. The degree-degree correlation plays a key role in determining the resiliency of the networks and can be measured by calculating assortativity $[7,32,53]$.

$$
r=\frac{E^{-1} \sum_{i} j_{i} k_{i}-\left[E^{-1} \sum_{i} \frac{1}{2}\left(j_{i}+k_{i}\right)\right]^{2}}{E^{-1} \sum_{i} \frac{1}{2}\left(j_{i}^{2}+k_{i}^{2}\right)-\left[E^{-1} \sum_{i} \frac{1}{2}\left(j_{i}+k_{i}\right)\right]^{2}}
$$


where $j_{i}$ and $k_{i}$ are the degrees of the nodes, and $E$ represents the total number of edges. For $\mathrm{r}=0$, there is no correlation; $\mathrm{r}<0$ means that the network is disassortative; and $\mathrm{r}>0$ shows that the network is assortative.

We obtained the epochs with the length of $20 \mathrm{~s}$ and extracted 504 features for each subject by utilizing seven graph theory features with 8 electrodes and 9 sets of EEG features in each state. The topographical EEG plot was drawn by using the MATLAB code derived from the code by Víctor Martínez-Cagigal [54].

\subsection{Statistical Analysis}

In our current work, we used a non-parametric Wilcoxon's rank sum test to assess the statistically significant network properties of middle-aged and elderly groups. The results are considered significant at $p<0.05$. The analysis was performed using Prism 9, Windows version (GraphPad software, San Diego, CA, USA).

\subsection{Classification Algorithms and Performance Measures}

In order to perform the classification, we used three classifiers: KNN, RF, and SVM. Three different classification approaches were tested to evaluate the classification model. Three classifiers were compared in terms of the classification performance parameters to select a suitable classifier for a provided EEG data set. The KNN classifier, also called the lazy learner algorithm, assumes similarity between available data and new data and assigns the most similar class [55]. The KNN algorithm calculates the distance by utilizing the distance measure, e.g., Euclidean and Manhattan distance measures. In this work, the Euclidean distance measure was used for $\mathrm{K}=1,3$, and 5 . The SVM is a commonly used linear classifier that utilizes the hyperplane technique to maximize the distance from the nearest training datapoint to easily identify classes [56]. The SVM can predict good accuracy based on one of its abilities to select a suitable kernel function. We used the Pearson VII function-based universal kernel function. The RF is an ensemble learning technique that utilizes the concept of bagging, constructs a collection of decision trees, and takes the average to predict the output. The RF classifier builds a forest based on uncorrelated trees by using decision tree learning, and it is useful for both regression and classification tasks [57]. The graph theory features extracted from the EEG of both groups were used as input for the classifiers. Classification was performed for eyes-open, eyes-closed, and WM tasks. A normalization technique was also used to improve the performance of the classifiers. Classification was performed using WEKA software (Version 3.8.4, Waikato University, New Zealand).

We used 10-fold cross validation to evaluate the classification models. The performance of classifiers is evaluated with overall accuracy (Acc), sensitivity (Sen), specificity (Spe), Kappa statistics (Ks), precision, and F-score, as shown in Equations (10)-(15).

$$
\begin{gathered}
\text { Acc }=\frac{(\mathrm{TP}+\mathrm{TN})}{(\mathrm{TP}+\mathrm{TN}+\mathrm{FP}+\mathrm{FN})} \times 100 \% \\
\text { Sen }=\frac{\mathrm{TP}}{\mathrm{TP}+\mathrm{FN}} \times 100 \% \\
\mathrm{Spe}=\frac{\mathrm{TN}}{\mathrm{TN}+\mathrm{FP}} \times 100 \% \\
\mathrm{Ks}=\frac{\mathrm{P}_{\mathrm{a}}-\mathrm{P}_{\mathrm{b}}}{1-\mathrm{P}_{\mathrm{b}}} \\
\mathrm{F}-\text { Score }=\frac{2 \times \mathrm{TP}}{(2 \times \mathrm{TP}+\mathrm{FP}+\mathrm{FN})} \times 100 \% \\
\text { Precision }=\frac{\mathrm{TP}}{\mathrm{TP}+\mathrm{FP}} \times 100 \%
\end{gathered}
$$


where TP is a true positive, $\mathrm{TN}$ is a true negative, FP is a false positive, and $\mathrm{FN}$ is a false negative. $\mathrm{P}_{\mathrm{a}}$ shows the observed proportion of agreement, and $\mathrm{P}_{\mathrm{b}}$ indicates the proportion of agreement expected by chance in Equation (13).

\section{Results}

We analyzed the network properties of the middle-aged and elderly groups during a resting state and while performing a WM task. Figure 2 presents a comparison of graph theory features of middle-aged vs. elderly individuals. Global efficacy, local efficiency, clustering coefficient, and node strength were found to be significantly lower in elderly subjects in the eyes-open state. In the eyes-closed state, all investigated parameters are shown in Figure 3, including global efficiency, local efficiency, characteristic path length, clustering coefficient, and node strength. Significant changes in global efficiency, local efficiency, clustering coefficient, and node strength were found to be lower in elderly subjects, but not characteristic path length. In a WM state, six network features showed significant changes, including global efficiency, local efficiency, characteristic path length clustering coefficient, assortativity, and node strength, as presented in Figure 4. Compared to the middle-aged group, the elderly group showed decreased network properties in all six significant features. Local efficiency, global efficiency, and clustering coefficient were significant in eyes-open, eyes-closed, and WM tasks.

a)

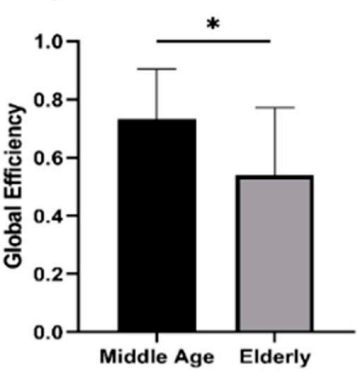

d)
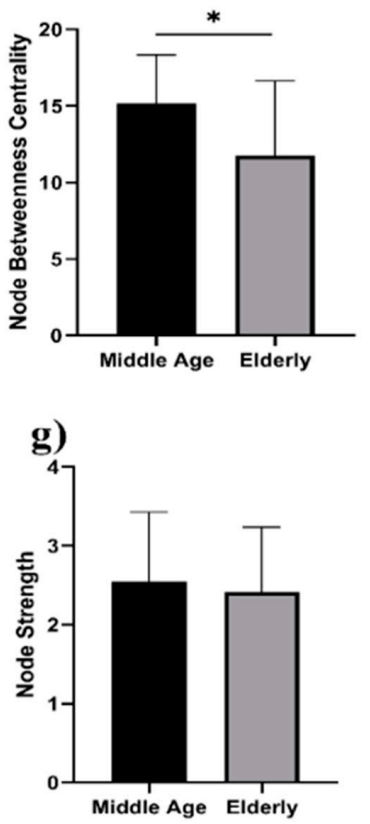

b)

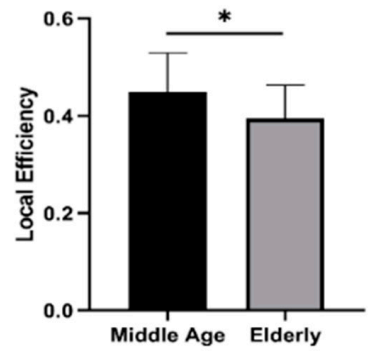

e)

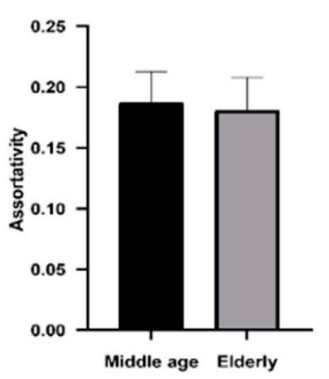

c)
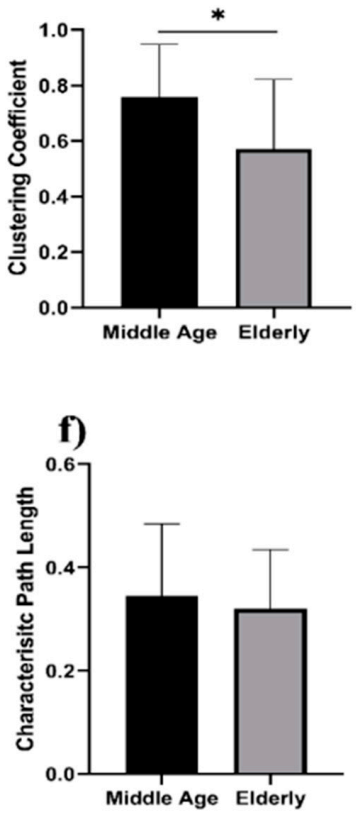

Figure 2. Eyes-open in middle-aged vs. elderly subjects' network features analysis (Wilcoxon's test; * $p<0.05$ ): (a) global efficiency, (b) local efficiency, (c) clustering coefficient, (d) node betweenness centrality, (e) assortativity, (f) characteristic path length, and (g) node strength. 
a)

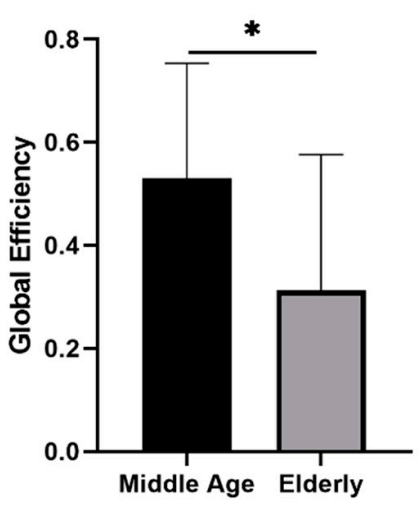

d)

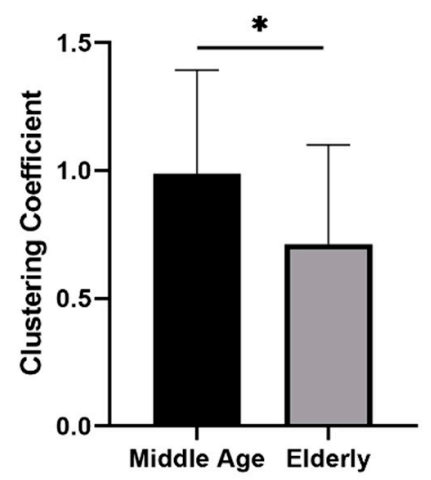

g)

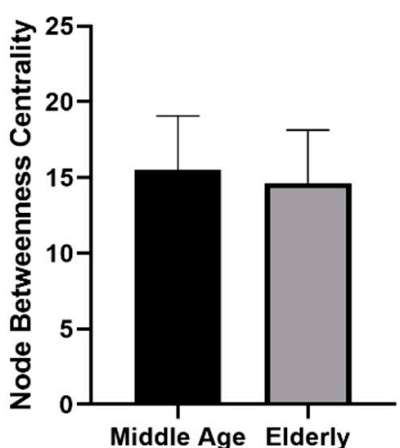

b)

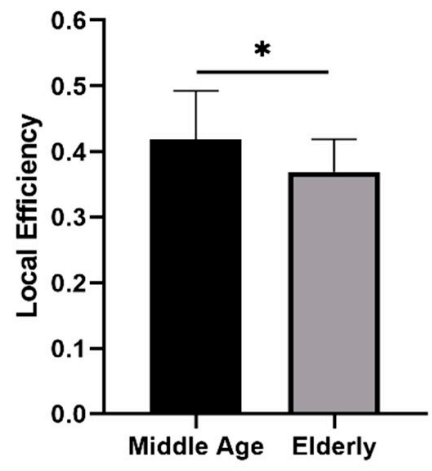

e)

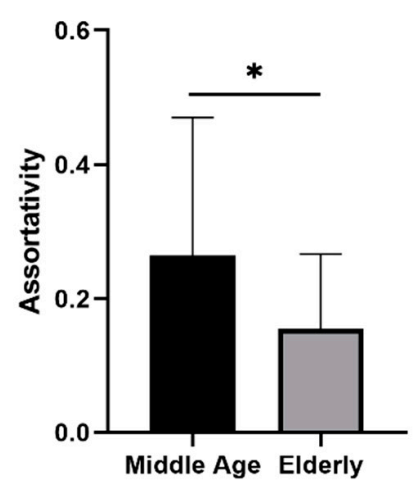

c)

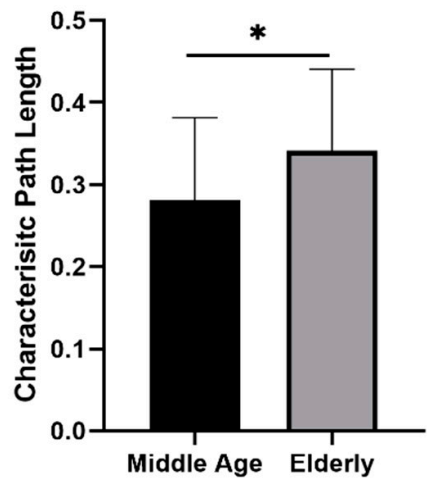

f)

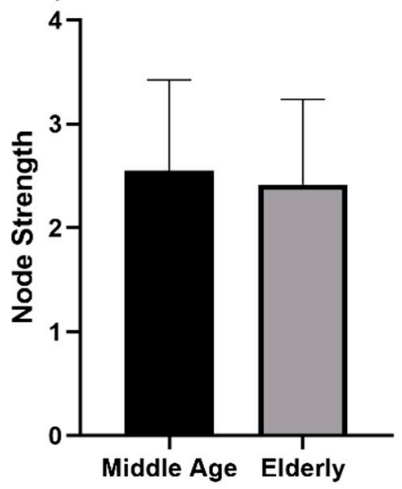

Figure 3. Eyes-closed in middle-aged vs. elderly individuals' network features analysis (Wilcoxon's test; ${ }^{*} p<0.05$ ): (a) global efficiency, (b) local efficiency, (c) characteristic path length, (d) clustering coefficient, (e) assortativity, (f) node strength, and (g) node betweenness centrality.

Significant changes in global efficiency, local efficiency, clustering coefficient, and node strength were found lower in the elderly subjects, but not characteristic path length. In the visual WM task, six network features showed significant changes, including global efficiency, local efficiency, characteristic path length clustering coefficient, assortativity, and node strength, as presented in Figure 4. Compared to the middle-aged group, the elderly group showed decreased network properties in all six significant features. Local efficiency, global efficiency, and clustering coefficient were significant in eyes-open, eyes-closed, and visual WM tasks. 
a)

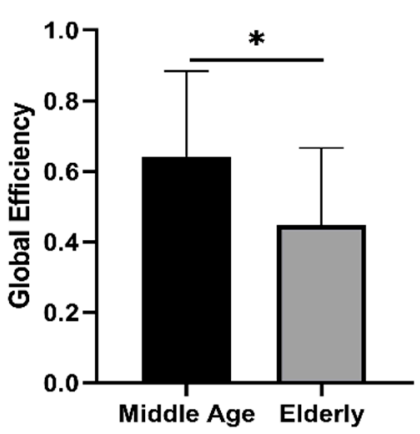

d)

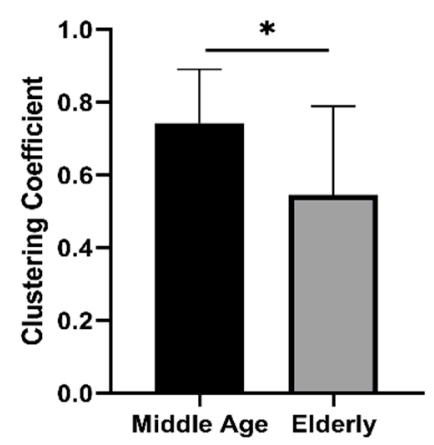

g)

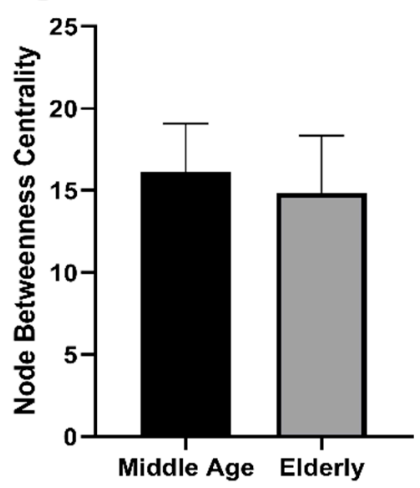

b)

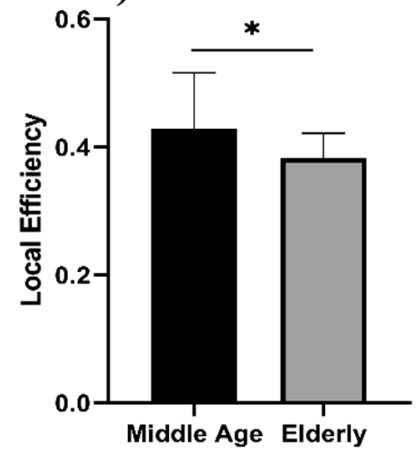

e)

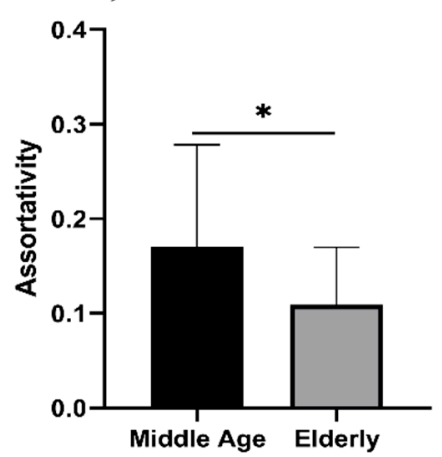

c)

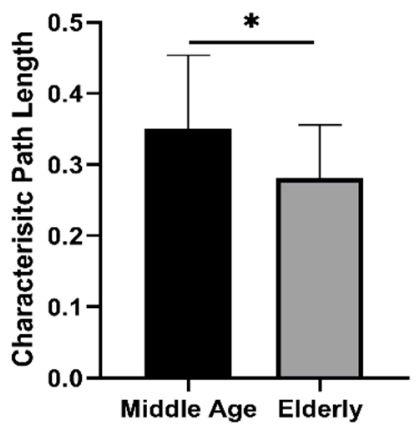

f)

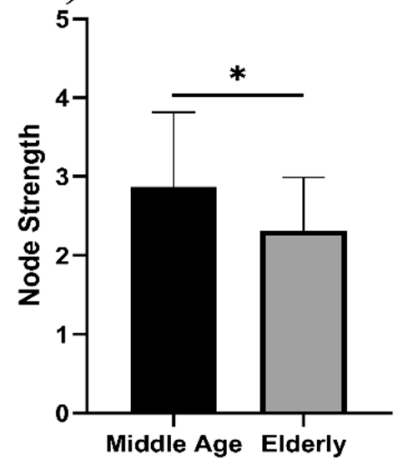

Figure 4. WM task in middle-aged vs. elderly individuals' network features analysis (Wilcoxon's test; * $p<0.05)$ : (a) global efficiency, (b) local efficiency, (c) characteristic path length, (d) clustering coefficient, (e) assortativity, (f) node strength, and (g) node betweenness centrality.

We used three classification algorithms to classify the middle-aged and elderly EEG, and the extracted features were used as input for these classifiers. KNN achieved its highest accuracy in the resting state (eyes open and eye closed) and during the WM task to classify the EEG signals obtained from middle-aged and elderly groups. KNN achieved $87.80 \%$ accuracy for $\mathrm{K}=3$ with a Euclidean distance measure in an eyes-open state, $93.33 \%$ accuracy with $\mathrm{K}=3$ in an eyes-closed state, and $98.89 \%$ accuracy for $\mathrm{K}=5$ in the WM task, as shown in Tables 1-3. The value of $\mathrm{K}$ in $\mathrm{KNN}$ was evaluated for 1, 3, and 5. In an eyes-open state for $\mathrm{K}=185.50 \%, \mathrm{~K}=5$ achieved $76.66 \%$ accuracy. In an eyes-closed state, we achieved an accuracy of $91.11 \%$ with $\mathrm{K}=1$ and achieved $87.77 \%$ accuracy for $\mathrm{K}=5$. In the visual WM task, classification accuracies of $94.40 \%$ and $97.78 \%$ were obtained for $\mathrm{K}=1$ and $\mathrm{K}=3$, respectively. 
Table 1. Classification parameter results in the eyes-open state.

\begin{tabular}{cccccc}
\hline & Accuracy (\%) & Sensitivity & Specificity & Kappa Statistics & F-Score \\
\hline KNN & 87.80 & 0.927 & 0.829 & 0.756 & 0.878 \\
RF & 85.36 & 0.902 & 0.805 & 0.707 & 0.853 \\
SVM & 86.67 & 0.911 & 0.822 & 0.733 & 0.866 \\
\hline
\end{tabular}

Table 2. Classification parameter results in the eyes-closed state.

\begin{tabular}{cccccc}
\hline & Accuracy (\%) & Sensitivity & Specificity & Kappa Statistics & F-Score \\
\hline KNN & 93.33 & 0.956 & 0.911 & 0.867 & 0.935 \\
RF & 91.11 & 0.889 & 0.933 & 0.822 & 0.911 \\
SVM & 91.10 & 0.978 & 0.844 & 0.822 & 0.911 \\
\hline
\end{tabular}

Table 3. Classification parameter results in the visual WM task.

\begin{tabular}{cccccc}
\hline & Accuracy (\%) & Sensitivity & Specificity & Kappa Statistics & F-Score \\
\hline KNN & 98.89 & 0.978 & 0.998 & 0.956 & 0.989 \\
RF & 97.78 & 0.980 & 0.976 & 0.889 & 0.978 \\
SVM & 94.44 & 0.956 & 0.933 & 0.978 & 0.944 \\
\hline
\end{tabular}

Figure 5 shows the topographical map of mean global efficiency, local efficiency, and mean clustering coefficient values of middle-aged and elderly groups in the eyes-open state. All three network parameters show differences in the whole brain network in different brain regions. In the eyes-closed state, mean local and global efficiencies and clustering coefficients clearly show differences, as shown in Figure 6. During the visual WM task, differences can be seen in local efficiency, global efficiencyas well as in clustering coefficient, as shown in Figure 7.

Table 1 shows the good sensitivity and specificity values of $\mathrm{KNN}$, and the highest Kappa value of 0.756 and an F-score of 0.878 confirm better performance in an eyes-open state. In an eyes-closed state, the highest Kappa value of 0.867 and an F-score of 0.935 confirm the best performance of KNN, as shown in Table 2. In the visual WM task, KNN obtained an excellent Kappa value of 0.956 and an F-score of 0.989 (Table 3). The sensitivity and specificity measures in the visual WM task validate the overall excellent performance of the classification model based on KNN.

Table 4 shows the weighted average precision and the area under the curve (AUC) for ROC curve of all classifiers in resting and visual working memory task states. KNN achieved the highest precision value of 0.989 during the visual WM task, while the highest AUC for ROC curve was achieved by RF (AUC $=0.988$ ), followed by KNN with 0.979 .

Table 4. Results of classification and performance of all classifiers.

\begin{tabular}{ccccccc}
\hline \multirow{2}{*}{ Activity } & \multicolumn{3}{c}{ Precision } & \multicolumn{3}{c}{ AUC } \\
\cline { 2 - 7 } & KNN & RF & SVM & KNN & RF & SVM \\
\hline Eyes-Open & 0.883 & 0.858 & 0.870 & 0.878 & 0.952 & 0.867 \\
Eyes-Closed & 0.934 & 0.912 & 0.919 & 0.935 & 0.658 & 0.912 \\
Visual WM Task & 0.989 & 0.978 & 0.945 & 0.979 & 0.988 & 0.944 \\
\hline
\end{tabular}



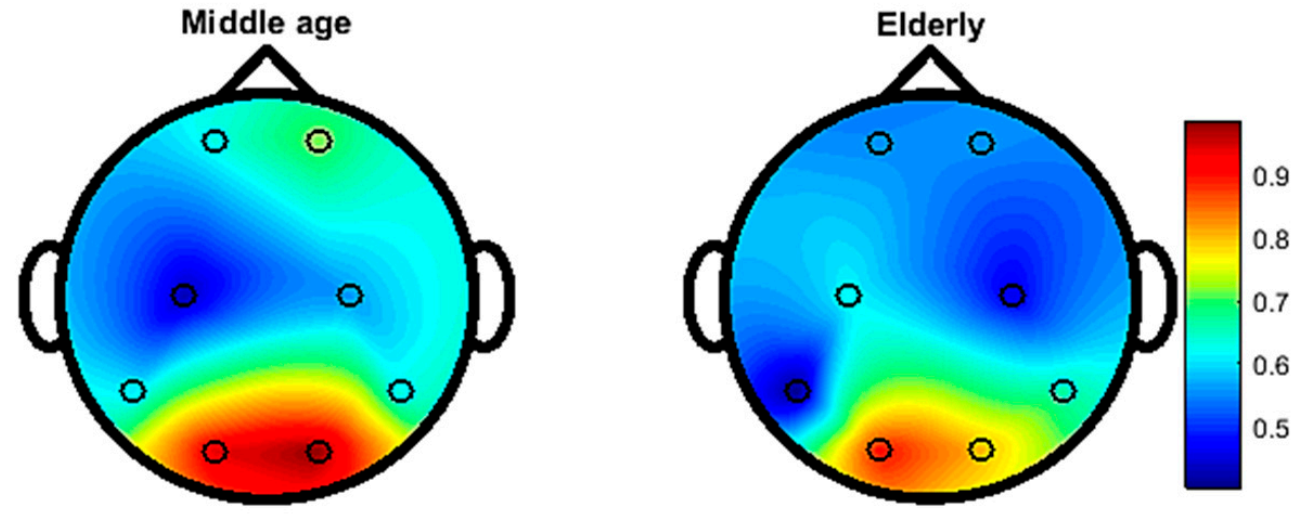

(a) Mean global efficiency in eyes-open state
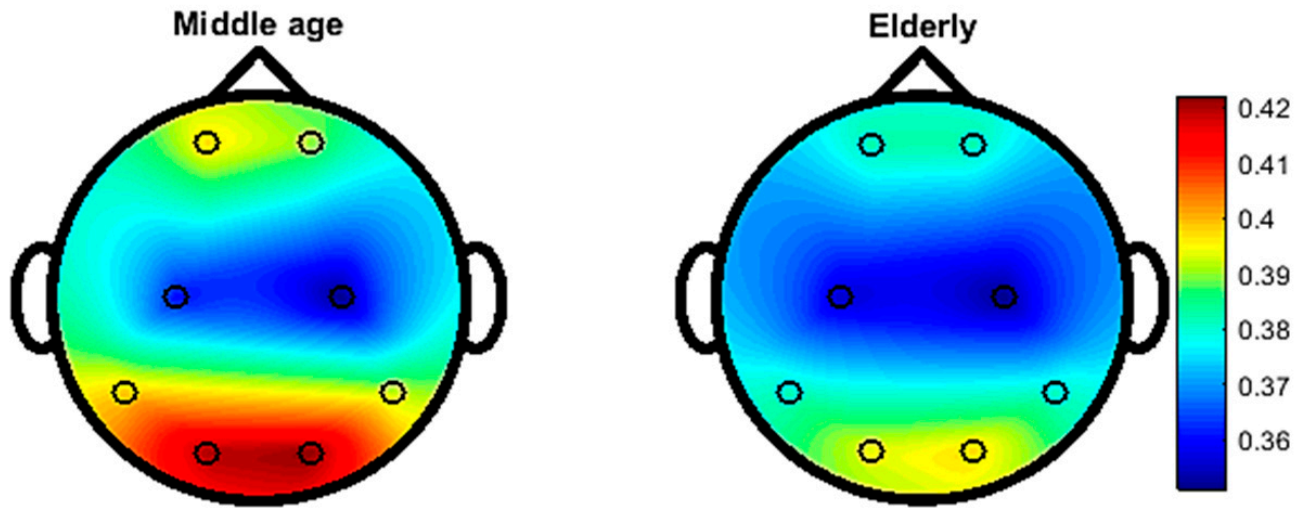

(b) Mean local efficiency in eyes-open state
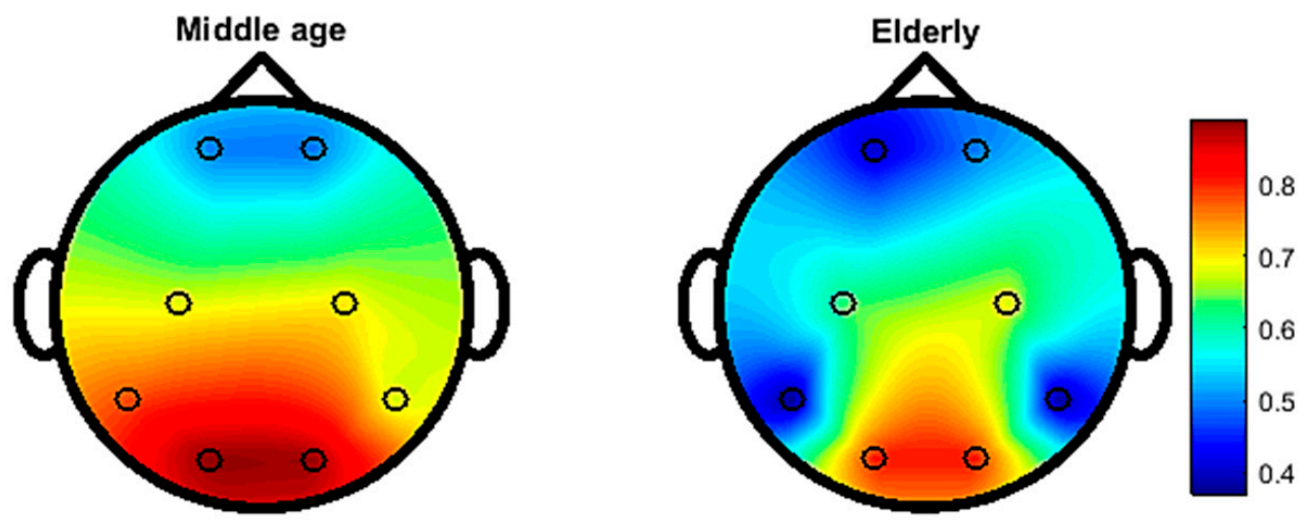

(c) Mean clustering coefficient in eyes-open state

Figure 5. Topographic map of EEG network features for middle-aged vs. elderly in eyes-open state: (a) mean global efficiency, (b) mean local efficiency, and (c) mean clustering coefficient.

The confusion matrices of all classifiers are presented for eyes-open, eyes-closed and visual WM task states in Figures 8-10, respectively. Furthermore, Figures 8-10 demonstrate the correctly classified and incorrectly classified instances. 

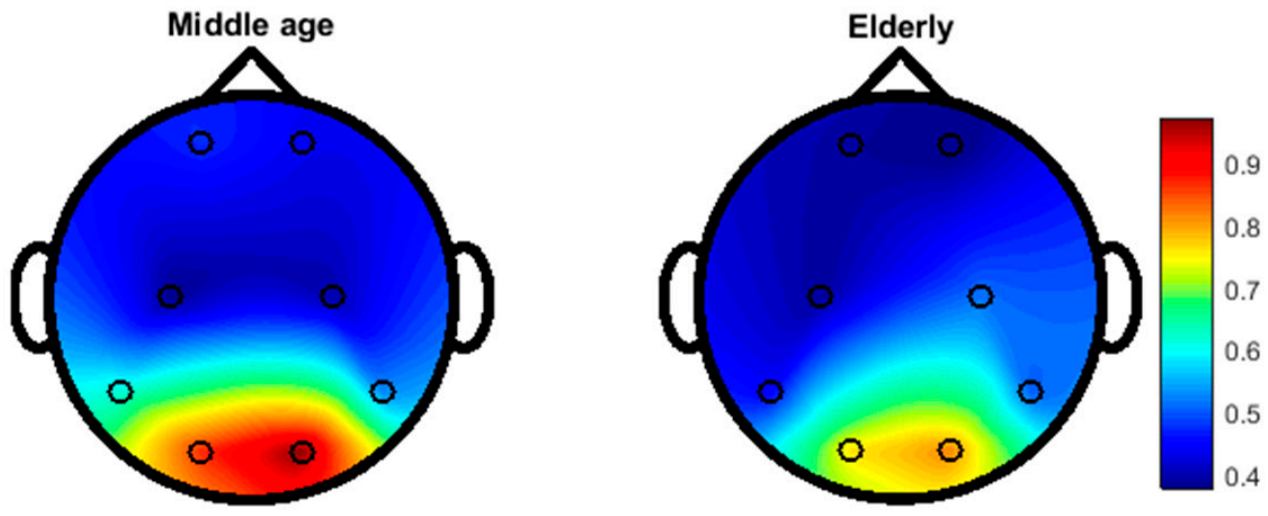

(a) Mean global efficiency in eyes-closed state
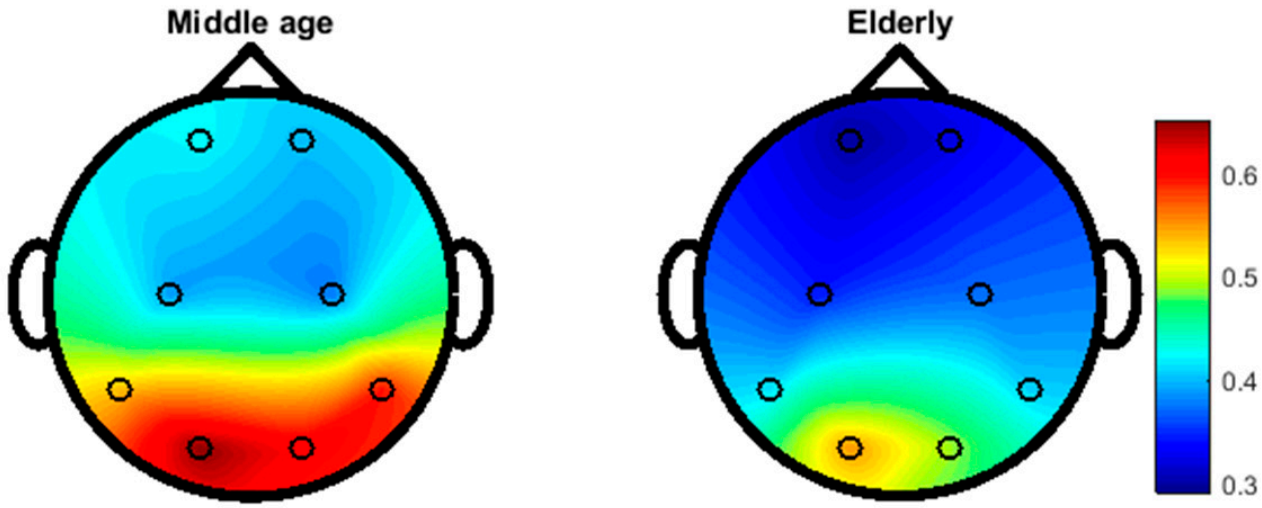

(b) Mean local efficiency in eyes-closed state
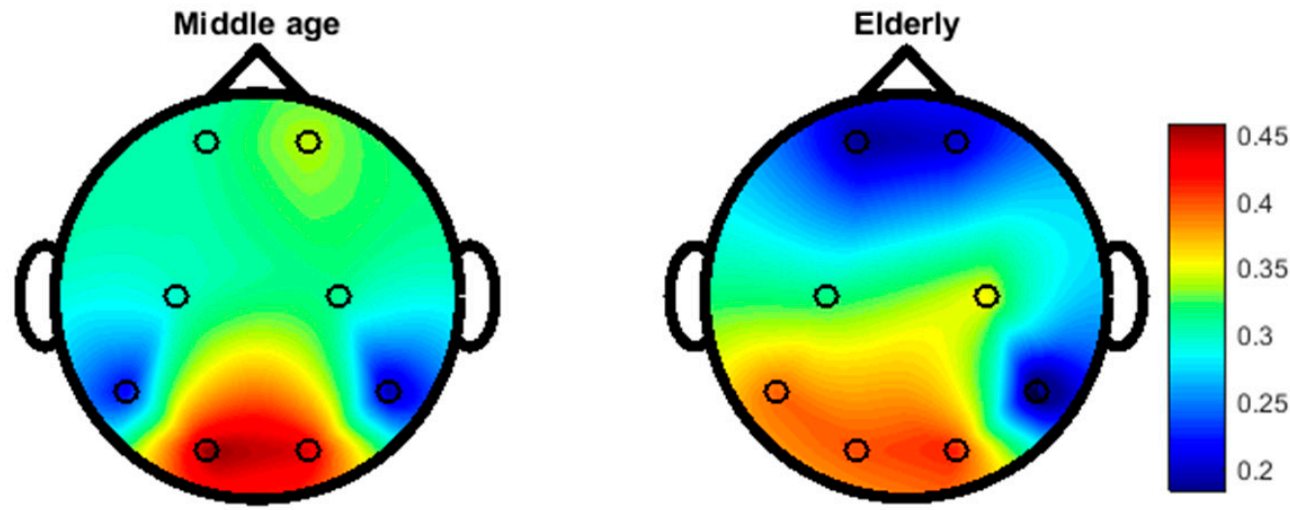

(c) Mean clustering coefficient in eyes-closed state

Figure 6. Topographic map of EEG network features for middle-aged vs. elderly in eyes-closed state: (a) mean global efficiency, (b) mean local efficiency, and (c) mean clustering coefficient.

The Figures 11-13 show the ROC plot of all three classifiers in eyes-open, eyes-closed and during the working memory task state. 

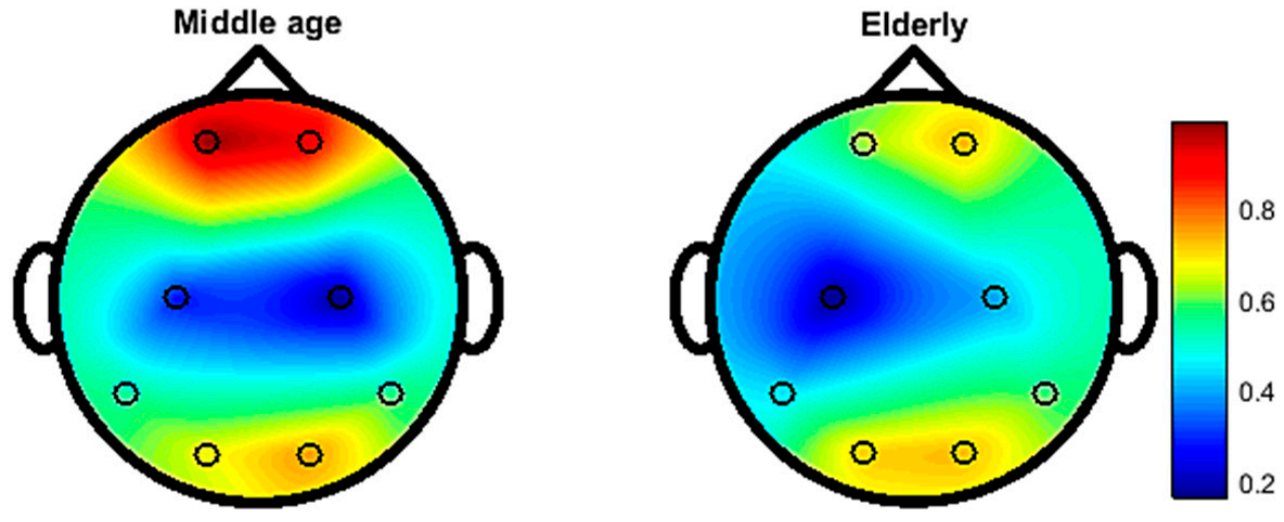

(a) Mean global efficiency in WM task state
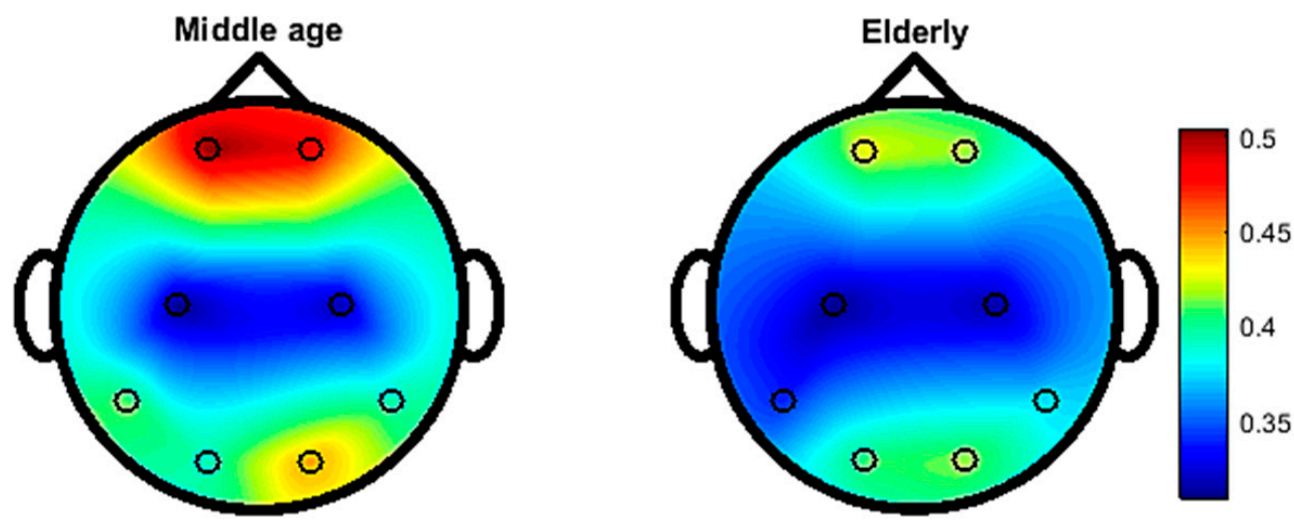

(b) Mean local efficiency in WM task state
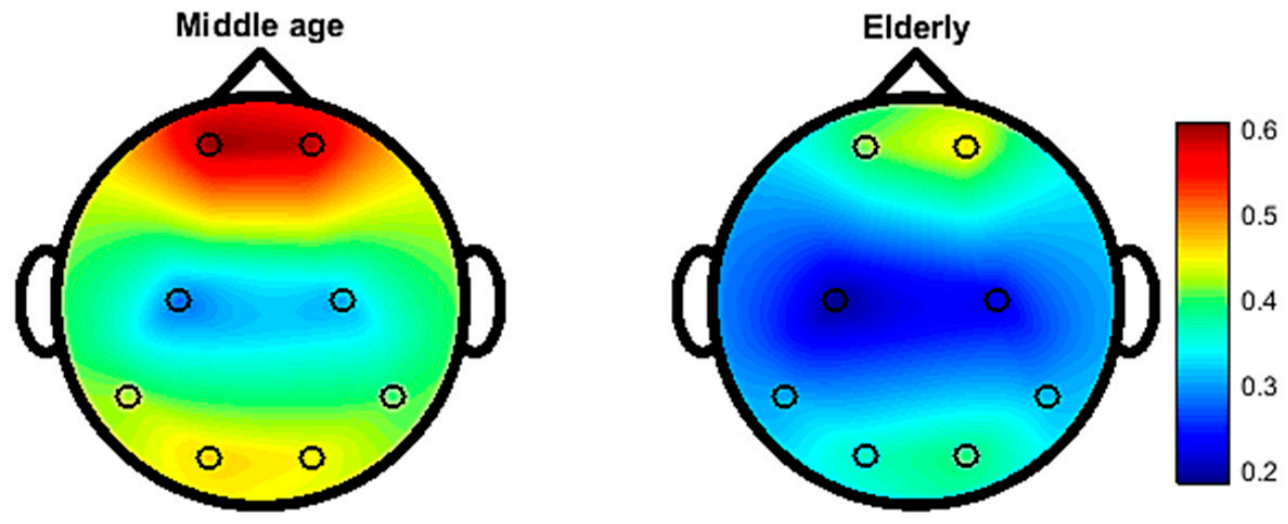

(c) Mean clustering coefficient in WM task state

Figure 7. Topographic map of EEG network features for middle-aged vs. elderly in the visual WM task state. (a) mean global efficiency, (b) mean local efficiency, and (c) mean clustering coefficient. 


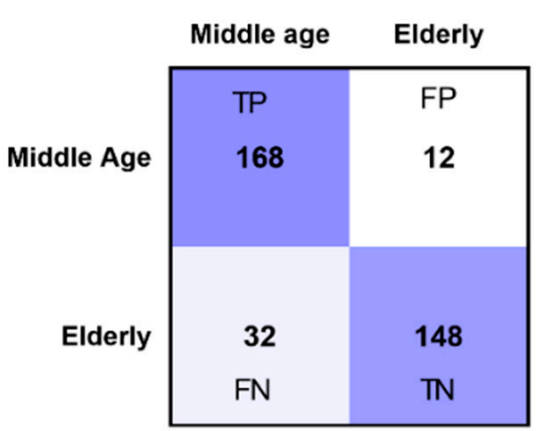

(a) Confusion Matrix of KNN

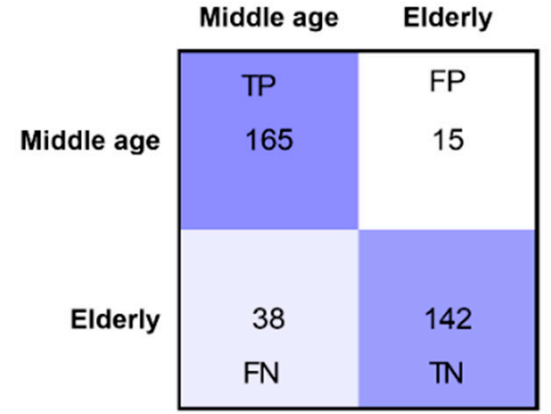

(b) Confusion Matrix of RF

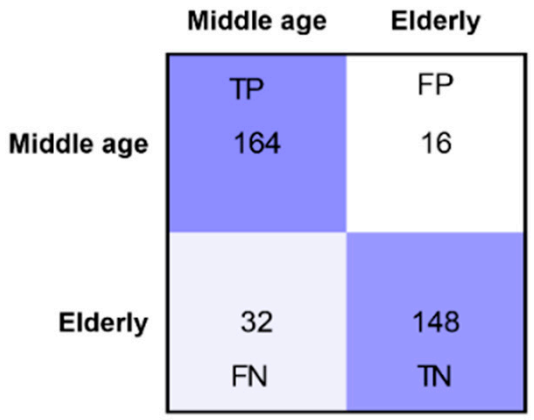

(c) Confusion Matrix of SVM

Figure 8. Confusion matrices of all classifiers in eyes-open state.

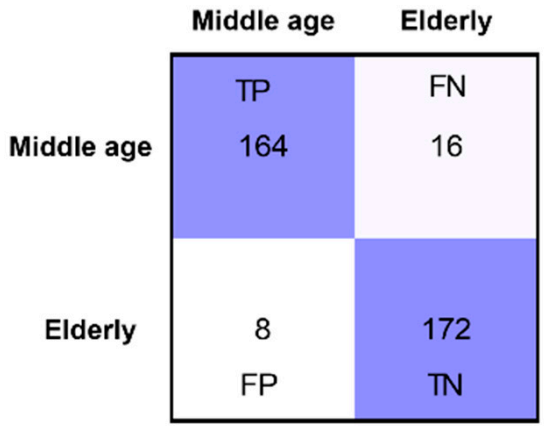

(a) Confusion Matrix of KNN

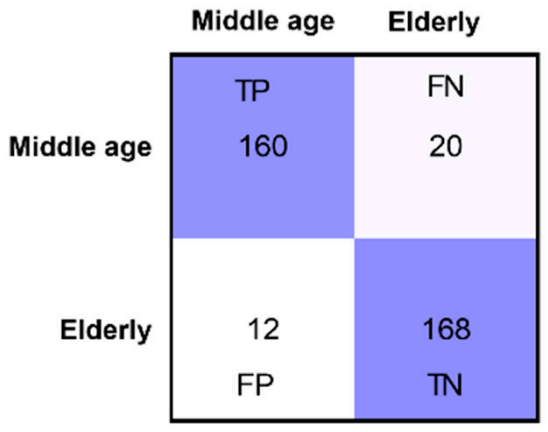

(b) Confusion Matrix of RF

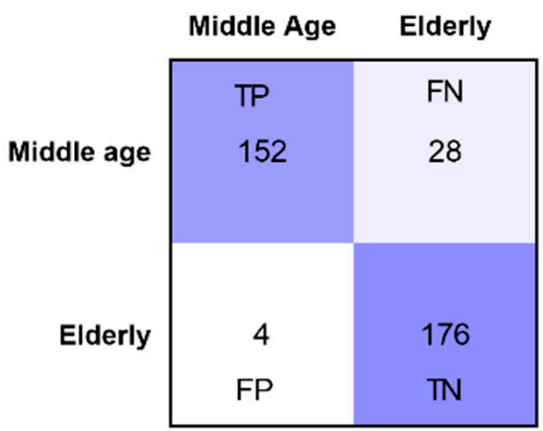

(c) Confusion Matrix of SVM

Figure 9. Confusion matrices of all classifiers in eyes-closed state. 


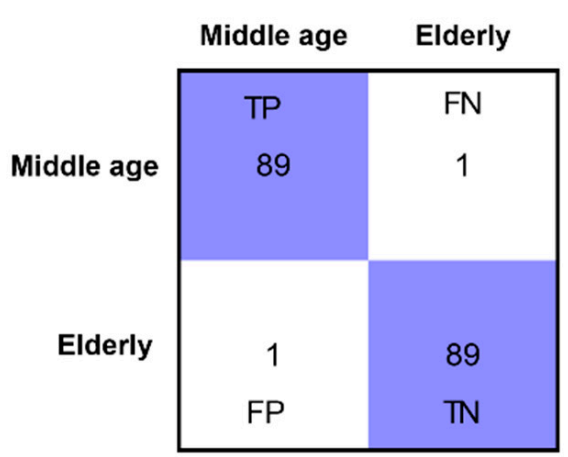

(a) Confusion Matrix of KNN

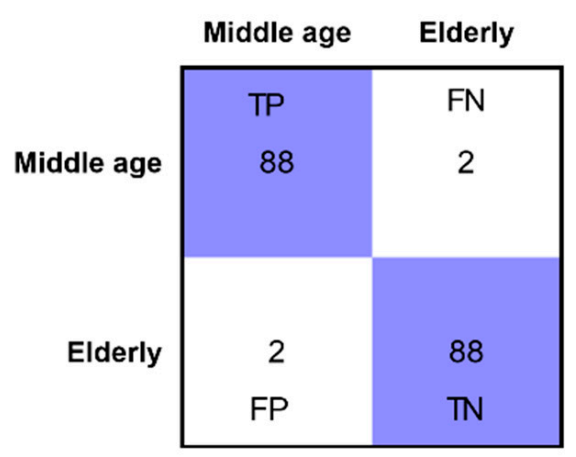

(b) Confusion Matrix of RF

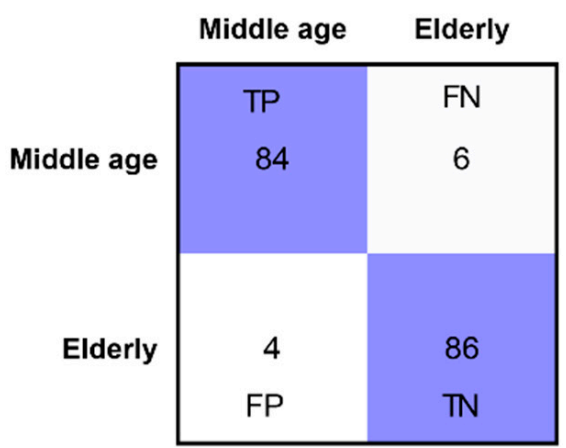

(c) Confusion Matrix of SVM

Figure 10. Confusion matrices of all classifiers in WM task state.

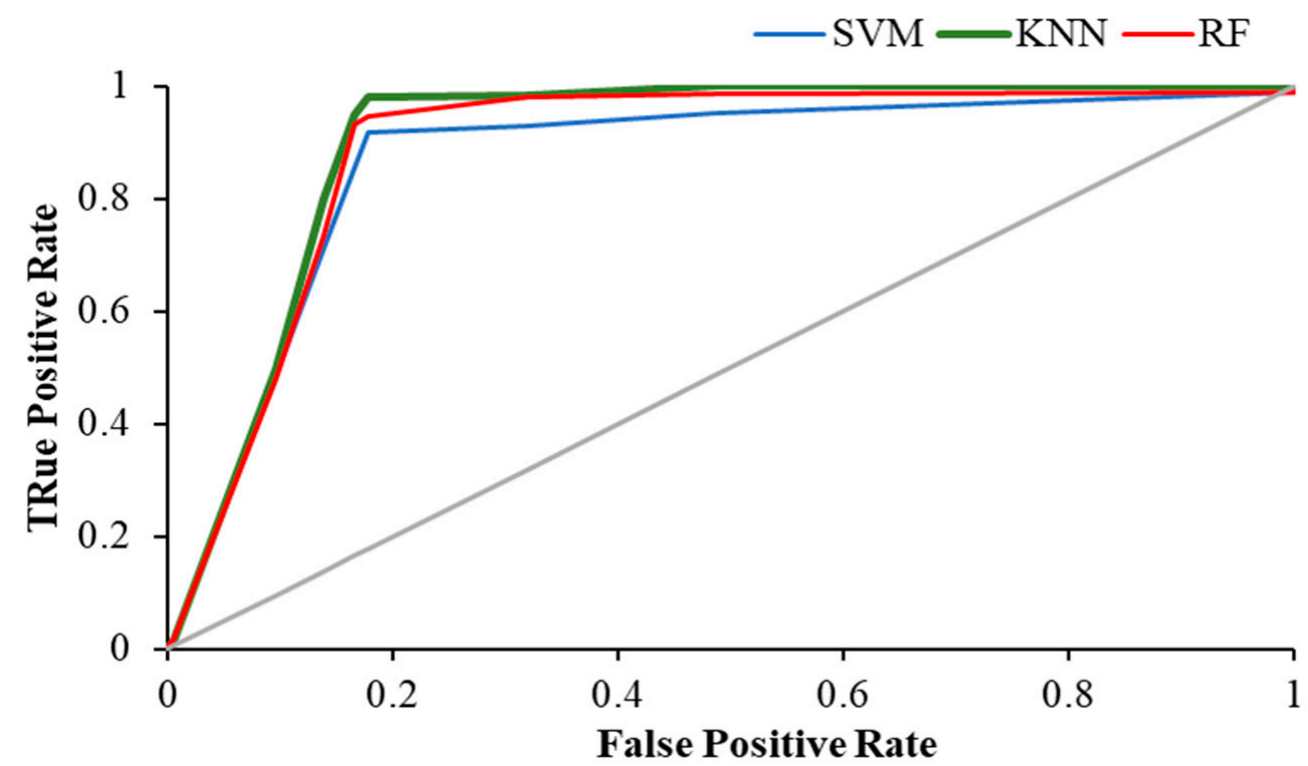

Figure 11. ROC plot of all classifiers in eyes-open state. 


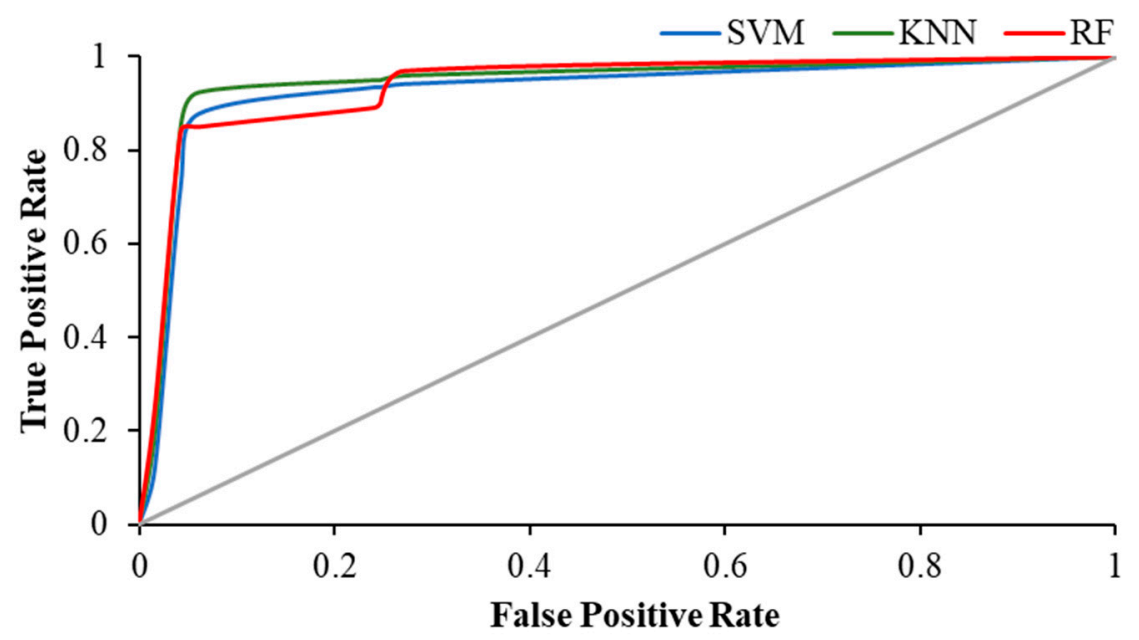

Figure 12. ROC plot of all classifiers in eyes-closed state.

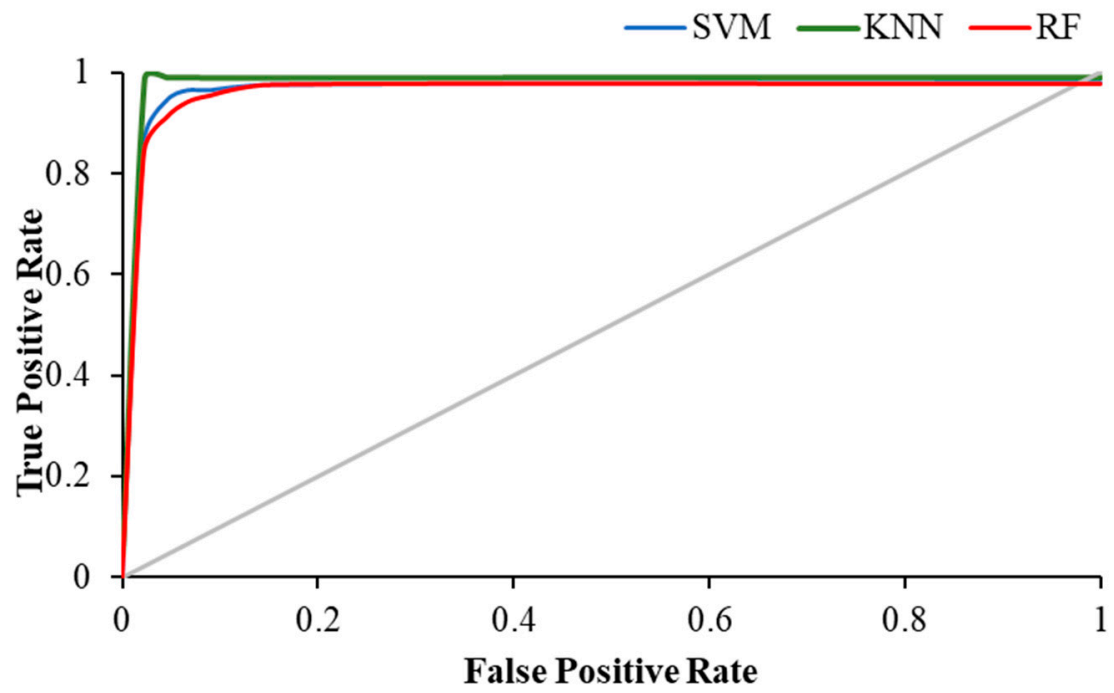

Figure 13. ROC plot of all classifiers in WM task state.

\section{Discussion}

In this work, a middle-aged group was compared with an elderly group in eyes-open and eyes-closed states, as well as during a visual WM task, to determine network matrices. Several network features were calculated from all subjects, and a statistical analysis was performed. The statistical analysis showed differences in a resting state and in working memory state networks. We further extended our described technique by combining the machine learning classification with three well-known classifiers (RF, KNN, and SVM). Our classification model achieved $98.89 \%$ accuracy with KNN during a WM task, thus corroborating the efficacy of our technique using EEG network features. In a resting state, the eyes-closed state of KNN achieved 93.33\% accuracy, which was higher than in the eyes-open state.

Our results indicate reduced network characteristics in terms of local efficiency, global efficiency, clustering coefficients, and node betweenness centrality in a resting state. In line with our work, a recent study found decreased local and global efficiency and clustering coefficients in middle-aged subjects compared to young adults [6]. In the previous study, a decrease in clustering coefficients confirmed age-related differences in oscillatory networks in young adults and elderly individuals, thus reinforcing our findings [18]. An increased path length indicates a less organized network characterized by less power in older adults, which confirms our results for the eyes-closed state [11]. With the eyes open, functional 
connectivity was decreased in alpha and beta frequency bands [58,59]. The opening or closing of the eyes can result in the functional connectivity of the brain being turned into an interoceptive or exteroceptive state [60]. Evidently, eyes-open and eyes-closed states have an impact on brain functional connectivity and network communication, implying that aging can generate more random brain networks in line with eyes-open and eyesclosed states $[18,61,62]$. Furthermore, our results confirm age-related differences between middle-aged and elderly individuals in terms of oscillatory networks

Our results in Figures 5 and 6 show the transition from middle-aged to older age in the configuration of brain network topology in a resting state, which is in line with the previous literature [6,62]. In healthy aging and an eyes-closed state, the global and lobal efficiency and clustering coefficient decreased with increasing age, in accordance with our study results (Figure 6) [6]. Our study shows that significant differences in occipital networks in older adults corroborate previous findings in a healthy aging, resting state network [18]. Working memory is considered to be dependent on a functional brain network to communicate efficiently and maintain a degree of modularity, and it can be disrupted by aging [63]. Additionally, our work shows a reduction in local and global efficiencies in a WM task, which is consistent with previous findings [64]. The age-related task induced changes, including a reduced clustering coefficient and network efficiency, in the elderly subjects, aligning our results with previous studies on performing a visual WM task [43]. Cognitive decline has been associated with structural and functional reorganization, with aging affecting cognitive performance in elderly populations $[65,66]$. WM has also been shown to be advantageous in increasing connectivity efficiency, and it is also useful for network reconfiguration to applying WM training in elderly subjects [43].

Neuroanatomical changes are considered to cause cognitive decline during the process of aging. We analyzed age-related differences in tasks that evoked alterations in EEG inter-regional synchronization in a healthy aging network topology. We conducted a crosssectional comparison of middle-aged and elderly subjects, and multichannel EEG was analyzed in a resting state and during a visual WM task. Compared to the resting state, the visual WM task showed more significant differences in terms of network measures. Local efficiency, global efficiency, clustering coefficient, characteristic path length, node strength, and assortativity were found to be found lower in elderly subjects. Age-related differences were reported during audiovisual processing using network topology parameters in a study in young vs. older adults [67]. In an n-back WM task, significant alterations were observed in older adults in network topology parameters such as clustering coefficient and path length, which supports our findings from the visual WM task [43]. Furthermore, we also found reduced assortativity in elderly compared to middle-aged subjects, and there was less resiliency among elderly networks compared to middle-aged networks.

The measure of assortativity showed significant differences in functional networks between healthy and AD networks, which confirms the efficacy of the assortativity measure in our work [32]. Network topology parameters were used in recent studies to distinguish between healthy elderly and AD subjects [68-70]. As far as we know, most recent studies focus on local and global efficiency clustering coefficients and path length to investigate age-related differences in functional brain networks. Hence, our work proposed to examine the efficacy of node strength betweenness centrality, and assortativity to analyze age-related differences in connectivity and network topology of the human brain. Our study provides additional support for the investigation of the effect of natural aging on memory by utilizing the proposed network features.

It has been suggested that the band works as a communication mechanism in cortical areas [71]. Beta band activity plays a vital role in inter-neuronal communication in functional networks in working memory [72]. The beta band is associated with attention deficiency in elderly individuals in terms of working memory [73,74], and task-evoked alterations were observed in a beta band network topology $[43,73]$. A recent study suggested that age-related alterations in EEG network connectivity are correlated with age-related slowing in attention tasks [42]. Therefore, our results of network indices under a visual 
working memory task are in line with those of previous studies. Furthermore, our classification results reinforce the usefulness of machine learning techniques to distinguish between age-related differences in aging brain networks.

The graph indices show significant differences in the efficiency and clustering of networks in the resting state, as well as in the WM state. We further used these network features for the classification of middle-aged and elderly subjects. Three classifiers (KNN, $\mathrm{RF}, \mathrm{SVM}$ ) were used to evaluate the classification problem of middle-aged vs. elderly individuals. In an eyes-closed resting condition, to classify the young vs. middle-aged subjects, SVM achieved an accuracy greater than $82 \%$ using graph theory features. In our study, we obtained an accuracy of $87.80 \%$ in the eyes-open state and an accuracy of 93.33\% in the eyes-closed state using KNN. Our study shows excellent values of sensitivity, specificity, Kappa statistics, and F-measure values of KNN (Tables 1 and 2).

Table 5 shows previous work based on graph theory features and the application of machine learning classification using EEG in different scenarios. Our classification result shows improved classification accuracy, which also corroborates the efficacy of the features. In our work, the KNN algorithm achieved the highest accuracy compared to the previous studies in which SVM was applied. To distinguish between younger and older adult brain networks, using functional connectivity in a resting state, SVM obtained an accuracy of $94 \%$ in classifying the brain by age group [75]. In the present work, the highest classification accuracy was $98.89 \%$ with KNN during a visual WM task state when classifying middle-aged and elderly EEG brain networks (Table 3). We also obtained a higher classification accuracy during the WM task than in the resting state. It is clear from the classification performance measures that the KNN classifier performed better compared to SVM and RF. The best sensitivity and specificity achieved by KNN confirm the validity of the classification model. Our classification results clearly exhibit increased accuracy in the WM task. To the best of our knowledge, the performance of classification measures confirms the robustness of our methodology, as well as the highest accuracy obtained with $\mathrm{KNN}$ in the classification of brain aging using a graph theory network. The age-related differences in EEG networks reflect the process of normal aging. However, the absence of age-dependent changes in elderly individuals can probably render them vulnerable to cognitive decline, dementia, and AD. This study highlighted the potential of a working memory-based technique to evaluate age-related alterations in a functional brain network and its associated mechanism, thus affecting memory in elderly populations.

Table 5. Comparison of current work with related EEG studies.

\begin{tabular}{|c|c|c|c|c|}
\hline Study & Application & State & Features & Classification Results \\
\hline Jalili, M [32] & $\begin{array}{l}\text { Alzheimer's disease } \\
\text { (AD vs. healthy) }\end{array}$ & $\begin{array}{l}\text { Resting state } \\
\text { (Eyes-open and } \\
\text { eyes-closed) }\end{array}$ & $\begin{array}{l}\text { Local efficiency, transitivity, } \\
\text { global efficiency, node and } \\
\text { edge between centrality, } \\
\text { assortativity, and modularity }\end{array}$ & $\mathrm{SVM}=82 \%$ \\
\hline Bahar Moezzi et al. [75] & $\begin{array}{l}\text { Healthy aging } \\
\text { (young vs. old) }\end{array}$ & $\begin{array}{l}\text { Resting state } \\
\text { (Eyes-open) }\end{array}$ & $\begin{array}{c}\text { Power spectra, functional } \\
\text { connectivity, and } \\
\text { electrode-to- } \\
\text { electrode distance }\end{array}$ & $\mathrm{SVM}=94 \%$ \\
\hline Petti, Manuela et al. [6] & $\begin{array}{c}\text { Healthy aging } \\
\text { (young vs. middle-aged) }\end{array}$ & $\begin{array}{l}\text { Resting state } \\
\text { (Eyes-closed) }\end{array}$ & $\begin{array}{l}\text { Node strength, local } \\
\text { efficiency, global efficiency, } \\
\text { clustering coefficient, } \\
\text { weight, and characteristic } \\
\text { path length }\end{array}$ & $\mathrm{SVM}=82 \%$ \\
\hline Lotfan, Saeed et al. [76] & $\begin{array}{l}\text { Social stress measurement } \\
\text { (healthy young) }\end{array}$ & $\begin{array}{l}\text { Before, right after, and } \\
20 \text { min after stress }\end{array}$ & $\begin{array}{l}\text { Transitivity, modularity, } \\
\text { characteristic path length, } \\
\text { and global efficiency }\end{array}$ & $\mathrm{SVM}=84.14 \%$ \\
\hline Proposed work & $\begin{array}{c}\text { Healthy aging } \\
\text { (middle-aged vs. elderly) }\end{array}$ & $\begin{array}{l}\text { Eyes-open, eyes-closed, } \\
\text { and visual WM task }\end{array}$ & $\begin{array}{l}\text { Global efficiency, local } \\
\text { efficiency, clustering } \\
\text { coefficient, characteristic } \\
\text { path length, node strength, } \\
\text { and assortativity }\end{array}$ & $\mathrm{KNN}=98.89 \%$ \\
\hline
\end{tabular}




\section{Conclusions}

This study presents a method for investigating the differences in elderly and middleaged EEG functional networks in eyes-open and eyes-closed states, as well as during a simple working memory task. Seven graph theory features were used, including local efficiency, global efficiency, clustering coefficient, characteristic path length, node strength, node betweenness centrality, and assortativity. Our analysis showed significant differences in both resting and working memory states. The seven network features were utilized as inputs for the classification models to distinguish between middle-aged and elderly EEG networks. In a resting state, a maximum accuracy of $93.33 \%$ was obtained in the eyes-closed state using KNN. In addition, KNN obtained the highest accuracy of $98.89 \%$ in the WM task state, with all seven features used in this study. The local and global efficiency, as well as the clustering coefficient, were the common features showing significant results in the eyes-open and eyes-closed states, as well as under a WM task condition. Our findings underscore the efficacy of working memory in investigating changes in brain network topology in relation to healthy aging. The eye blinks and eye movements in working memory may distort the results of coherence. Furthermore, in the future, the addition of young adults in our studies ( $<40$ years) and the increase of number of electrodes will clarify the impact of brain aging and age-related issues on pathological conditions such as mild cognitive impairment and the preclinical stages of Alzheimer's disease. The proposed technique can be extended further with advanced forms of research to develop a biomarker of aging using EEG signals.

Author Contributions: Conceptualization, H.J., E.K. and S.C.; formal analysis, H.J., E.K. and S.C.; methodology, H.J.; supervision, E.K. and S.C.; writing-original draft, H.J.; writing-review and editing, E.K. and S.C.; funding acquisition, E.K. All authors have read and agreed to the published version of the manuscript.

Funding: This work was financially supported by grants from Prince of Songkla University, Songkhla, Thailand (SCI6202110S).

Institutional Review Board Statement: The study was conducted according to the guidelines of the Declaration of Helsinki and approved by the Human Research Ethical Committee of Prince of Songkla University (HSC-HREC-61-006-02-1).

Informed Consent Statement: Informed consent was obtained from all subjects involved in the study.

Acknowledgments: We are grateful to the volunteers for their participation in this study. We are thankful to the Thailand's Education Hub for Southern Region of ASEAN Countries (TEH-AC) scholarship from the graduate school, Prince of Songkla University, offered to Hamad Javaid.

Conflicts of Interest: The authors declare no conflict of interest.

\section{References}

1. Bullmore, E.; Sporns, O. Complex brain networks: Graph theoretical analysis of structural and functional systems. Nat. Rev. Neurosci. 2009, 10, 186-198. [CrossRef] [PubMed]

2. Van den Heuvel, M.I.; Turk, E.; Manning, J.H.; Hect, J.; Hernandez-Andrade, E.; Hassan, S.S.; Romero, R.; van den Heuvel, M.P.; Thomason, M.E. Hubs in the human fetal brain network. Dev. Cogn. Neurosci. 2018, 30, 108-115. [CrossRef] [PubMed]

3. Micheloyannis, S.; Vourkas, M.; Tsirka, V.; Karakonstantaki, E.; Kanatsouli, K.; Stam, C.J. The influence of ageing on complex brain networks: A graph theoretical analysis. Hum. Brain Mapp. 2009, 30, 200-208. [CrossRef] [PubMed]

4. Smit, D.J.A.; Boersma, M.; Schnack, H.G.; Micheloyannis, S.; Boomsma, D.I.; Hulshoff Pol, H.E.; Stam, C.J.; de Geus, E.J.C. The brain matures with stronger functional connectivity and decreased randomness of its network. PLoS ONE 2012, 7, e36896. [CrossRef] [PubMed]

5. Gaál, Z.A.; Boha, R.; Stam, C.J.; Molnár, M. Age-dependent features of EEG-reactivity-Spectral, complexity, and network characteristics. Neurosci. Lett. 2010, 479, 79-84. [CrossRef]

6. Petti, M.; Toppi, J.; Babiloni, F.; Cincotti, F.; Mattia, D.; Astolfi, L. EEG resting-state brain topological reorganization as a function of age. Comput. Intell. Neurosci. 2016, 2016, 1-10. [CrossRef]

7. Rubinov, M.; Sporns, O. Complex network measures of brain connectivity: Uses and interpretations. Neuroimage 2010, 52, 1059-1069. [CrossRef] 
8. Betzel, R.F.; Fukushima, M.; He, Y.; Zuo, X.-N.; Sporns, O. Dynamic fluctuations coincide with periods of high and low modularity in resting-state functional brain networks. Neuroimage 2016, 127, 287-297. [CrossRef]

9. Zalesky, A.; Fornito, A.; Cocchi, L.; Gollo, L.L.; Breakspear, M. Time-resolved resting-state brain networks. Proc. Natl. Acad. Sci. USA 2014, 111, 10341-10346. [CrossRef]

10. Calhoun, V.D.; Miller, R.; Pearlson, G.; Adalı, T. The chronnectome: Time-varying connectivity networks as the next frontier in fMRI data discovery. Neuron 2014, 84, 262-274. [CrossRef]

11. Duffy, F.H.; Albert, M.S.; McAnulty, G.; Garvey, A.J. Age-related differences in brain electrical activity of healthy subjects. Ann. Neurol. 1984, 16, 430-438. [CrossRef] [PubMed]

12. Van der Hiele, K.; Bollen, E.L.E.M.; Vein, A.A.; Reijntjes, R.H.A.M.; Westendorp, R.G.J.; van Buchem, M.A.; Middelkoop, H.A.M.; van Dijk, J.G. EEG markers of future cognitive performance in the elderly. J. Clin. Neurophysiol. 2008, 25, 83-89. [CrossRef] [PubMed]

13. Bassett, D.S.; Wymbs, N.F.; Porter, M.A.; Mucha, P.J.; Carlson, J.M.; Grafton, S.T. Dynamic reconfiguration of human brain networks during learning. Proc. Natl. Acad. Sci. USA 2011, 108, 7641-7646. [CrossRef] [PubMed]

14. Davison, E.N.; Schlesinger, K.J.; Bassett, D.S.; Lynall, M.-E.; Miller, M.B.; Grafton, S.T.; Carlson, J.M. Brain network adaptability across task states. PLoS Comput. Biol. 2015, 11, e1004029. [CrossRef] [PubMed]

15. Chen, Y.; Zhao, X.; Zhang, X.; Liu, Y.; Zhou, P.; Ni, H.; Ma, J.; Ming, D. Age-related early/late variations of functional connectivity across the human lifespan. Neuroradiology 2018, 60, 403-412. [CrossRef] [PubMed]

16. Tian, L.; Li, Q.; Wang, C.; Yu, J. Changes in dynamic functional connections with aging. Neuroimage 2018, 172, 31-39. [CrossRef]

17. Zhang, J.; Cheng, W.; Liu, Z.; Zhang, K.; Lei, X.; Yao, Y.; Becker, B.; Liu, Y.; Kendrick, K.M.; Lu, G. Neural, electrophysiological and anatomical basis of brain-network variability and its characteristic changes in mental disorders. Brain 2016, 139, $2307-2321$. [CrossRef]

18. Knyazev, G.G.; Volf, N.V.; Belousova, L.V. Age-related differences in electroencephalogram connectivity and network topology. Neurobiol. Aging 2015, 36, 1849-1859. [CrossRef]

19. Roux, F.; Uhlhaas, P.J. Working memory and neural oscillations: Alpha-gamma versus theta-gamma codes for distinct WM information? Trends Cogn. Sci. 2014, 18, 16-25. [CrossRef]

20. Bopp, K.L.; Verhaeghen, P. Working memory and aging: Separating the effects of content and context. Psychol. Aging 2009, 24, 968. [CrossRef]

21. Li, H.-J.; Hou, X.-H.; Liu, H.-H.; Yue, C.-L.; Lu, G.-M.; Zuo, X.-N. Putting age-related task activation into large-scale brain networks: A meta-analysis of $114 \mathrm{fMRI}$ studies on healthy aging. Neurosci. Biobehav. Rev. 2015, 57, 156-174. [CrossRef] [PubMed]

22. Jacobs, E.G.; Weiss, B.; Makris, N.; Whitfield-Gabrieli, S.; Buka, S.L.; Klibanski, A.; Goldstein, J.M. Reorganization of functional networks in verbal working memory circuitry in early midlife: The impact of sex and menopausal status. Cereb. Cortex 2017,27, 2857-2870. [CrossRef] [PubMed]

23. Padgaonkar, N.A.; Zanto, T.P.; Bollinger, J.; Gazzaley, A. Predictive cues and age-related declines in working memory performance. Neurobiol. Aging 2017, 49, 31-39. [CrossRef] [PubMed]

24. Dong, S.; Reder, L.M.; Yao, Y.; Liu, Y.; Chen, F. Individual differences in working memory capacity are reflected in different ERP and EEG patterns to task difficulty. Brain Res. 2015, 1616, 146-156. [CrossRef]

25. Rypma, B.; D'Esposito, M. The roles of prefrontal brain regions in components of working memory: Effects of memory load and individual differences. Proc. Natl. Acad. Sci. USA 1999, 96, 6558-6563. [CrossRef]

26. Marvel, C.L.; Desmond, J.E. Functional topography of the cerebellum in verbal working memory. Neuropsychol. Rev. 2010, 20, 271-279. [CrossRef]

27. Vergauwe, E.; Hartstra, E.; Barrouillet, P.; Brass, M. Domain-general involvement of the posterior frontolateral cortex in time-based resource-sharing in working memory: An fMRI study. Neuroimage 2015, 115, 104-116. [CrossRef]

28. Toppi, J.; Astolfi, L.; Risetti, M.; Anzolin, A.; Kober, S.E.; Wood, G.; Mattia, D. Different topological properties of EEG-derived networks describe working memory phases as revealed by graph theoretical analysis. Front. Hum. Neurosci. 2018, 11, 637. [CrossRef]

29. Becker, H.; Fleureau, J.; Guillotel, P.; Wendling, F.; Merlet, I.; Albera, L. Emotion recognition based on high-resolution EEG recordings and reconstructed brain sources. IEEE Trans. Affect. Comput. 2017, 11, 244-257. [CrossRef]

30. Bansal, D.; Chhikara, R.; Khanna, K.; Gupta, P. Comparative analysis of various machine learning algorithms for detecting dementia. Procedia Comput. Sci. 2018, 132, 1497-1502. [CrossRef]

31. Hussain, I.; Park, S.-J. Quantitative evaluation of task-induced neurological outcome after stroke. Brain Sci. 2021, 11, 900. [CrossRef] [PubMed]

32. Jalili, M. Graph theoretical analysis of Alzheimer's disease: Discrimination of AD patients from healthy subjects. Inf. Sci. 2017, 384, 145-156. [CrossRef]

33. Yu, H.; Zhu, L.; Cai, L.; Wang, J.; Liu, J.; Wang, R.; Zhang, Z. Identification of Alzheimer's EEG With a WVG network-based fuzzy learning approach. Front. Neurosci. 2020, 14, 641. [CrossRef] [PubMed]

34. Pellegrini, E.; Ballerini, L.; Hernandez, M.d.C.V.; Chappell, F.M.; González-Castro, V.; Anblagan, D.; Danso, S.; Muñoz-Maniega, S.; Job, D.; Pernet, C.; et al. Machine learning of neuroimaging for assisted diagnosis of cognitive impairment and dementia: A systematic review. Alzheimer's Dement. Diagn. Assess. Dis. Monit. 2018, 10, 519-535. [CrossRef] [PubMed] 
35. Cassani, R.; Falk, T.H.; Fraga, F.J.; Cecchi, M.; Moore, D.K.; Anghinah, R. Towards automated electroencephalography-based Alzheimer's disease diagnosis using portable low-density devices. Biomed. Signal Process. Control 2017, 33, 261-271. [CrossRef]

36. Hussain, I.; Young, S.; Park, S.-J. Driving-induced neurological biomarkers in an advanced driver-assistance system. Sensors 2021, 21, 6985. [CrossRef]

37. Hussain, I.; Park, S.J. HealthSOS: Real-time health monitoring system for stroke prognostics. IEEE Access 2020, 8, $213574-213586$. [CrossRef]

38. Wang, X.; Gong, G.; Li, N.; Qiu, S. Detection analysis of epileptic EEG using a novel random forest model combined with grid search optimization. Front. Hum. Neurosci. 2019, 13, 52. [CrossRef]

39. Ota, M.; Koshibe, Y.; Higashi, S.; Nemoto, K.; Tsukada, E.; Tamura, M.; Takahashi, T.; Arai, T. Structural brain network correlated with reading impairment in Alzheimer's disease. Dement. Geriatr. Cogn. Disord. 2020, 49, 264-269. [CrossRef]

40. Vecchio, F.; Miraglia, F.; Quaranta, D.; Granata, G.; Romanello, R.; Marra, C.; Bramanti, P.; Rossini, P.M. Cortical connectivity and memory performance in cognitive decline: A study via graph theory from EEG data. Neuroscience 2016, 316, 143-150. [CrossRef]

41. Peláez Suárez, A.A.; Berrillo Batista, S.; Pedroso Ibáñez, I.; Casabona Fernández, E.; Fuentes Campos, M.; Chacón, L.M. EEGderived functional connectivity patterns associated with mild cognitive impairment in Parkinson's disease. Behav. Sci. 2021, 11, 40. [CrossRef] [PubMed]

42. Vecchio, F.; Miraglia, F.; Bramanti, P.; Rossini, P.M. Human brain networks in physiological aging: A graph theoretical analysis of cortical connectivity from EEG data. J. Alzheimer's Dis. 2014, 41, 1239-1249. [CrossRef] [PubMed]

43. Hou, F.; Liu, C.; Yu, Z.; Xu, X.; Zhang, J.; Peng, C.-K.; Wu, C.; Yang, A. Age-related alterations in electroencephalography connectivity and network topology during n-back working memory task. Front. Hum. Neurosci. 2018, 12, 484. [CrossRef] [PubMed]

44. Stam, C.J. Brain dynamics in theta and alpha frequency bands and working memory performance in humans. Neurosci. Lett. 2000, 286, 115-118. [CrossRef]

45. Autthasan, P.; Du, X.; Arnin, J.; Lamyai, S.; Perera, M.; Itthipuripat, S.; Yagi, T.; Manoonpong, P.; Wilaiprasitporn, T. A singlechannel consumer-grade EEG device for brain-computer interface: Enhancing detection of SSVEP and its amplitude modulation. IEEE Sens. J. 2019, 20, 3366-3378. [CrossRef]

46. Samson, V.R.R.; Praveen Kitti, B.; Pradeep Kumar, S.; Suresh Babu, D.; Monica, C. Electroencephalogram-based OpenBCI devices for disabled people. In Proceedings of 2nd International Conference on Micro-Electronics, Electromagnetics and Telecommunications; Springer: Berlin/Heidelberg, Germany, 2018; pp. 229-238.

47. Qiu, J.M.; Casey, M.A.; Diamond, S.G. Assessing feedback response with a wearable electroencephalography system. Front. Hum. Neurosci. 2019, 13, 258. [CrossRef]

48. Hinrichs, H.; Scholz, M.; Baum, A.K.; Kam, J.W.Y.; Knight, R.T.; Heinze, H.-J. Comparison between a wireless dry electrode EEG system with a conventional wired wet electrode EEG system for clinical applications. Sci. Rep. 2020, 10, 1-14. [CrossRef]

49. Delorme, A.; Makeig, S. EEGLAB: An open source toolbox for analysis of single-trial EEG dynamics including independent component analysis. J. Neurosci. Methods 2004, 134, 9-21. [CrossRef]

50. Jalili, M. Functional brain networks: Does the choice of dependency estimator and binarization method matter? Sci. Rep. 2016, 6 , 1-12. [CrossRef]

51. Achard, S.; Bullmore, E. Efficiency and cost of economical brain functional networks. PLoS Comput. Biol. 2007, 3, e17. [CrossRef]

52. Opsahl, T.; Agneessens, F.; Skvoretz, J. Node centrality in weighted networks: Generalizing degree and shortest paths. Soc. Netw. 2010, 32, 245-251. [CrossRef]

53. Newman, M.E.J. Assortative mixing in networks. Phys. Rev. Lett. 2002, 89, 208701. [CrossRef] [PubMed]

54. Martínez-Cagigal, V. Topographic EEG/MEG Plot. Available online: https://www.mathworks.com/matlabcentral/fileexchange/ 72729-topographic-eeg-meg-plot (accessed on 20 August 2021).

55. Qureshi, S.; Karrila, S.; Vanichayobon, S. Human sleep scoring based on K-nearest neighbors. Turk. J. Electr. Eng. Comput. Sci. 2018, 26, 2802-2818. [CrossRef]

56. Miltiadous, A.; Tzimourta, K.D.; Giannakeas, N.; Tsipouras, M.G.; Afrantou, T.; Ioannidis, P.; Tzallas, A.T. Alzheimer's disease and frontotemporal dementia: A robust classification method of EEG signals and a comparison of validation methods. Diagnostics 2021, 11, 1437. [CrossRef] [PubMed]

57. Breiman, L. Bagging predictors. Mach. Learn. 1996, 24, 123-140. [CrossRef]

58. Stam, C.J.; De Bruin, E.A. Scale-free dynamics of global functional connectivity in the human brain. Hum. Brain Mapp. 2004, 22, 97-109. [CrossRef]

59. Tan, B.; Kong, X.; Yang, P.; Jin, Z.; Li, L. The difference of brain functional connectivity between eyes-closed and eyes-open using graph theoretical analysis. Comput. Math. Methods Med. 2013, 2013, 1-15. [CrossRef]

60. Costumero, V.; Bueichekú, E.; Adrián-Ventura, J.; Ávila, C. Opening or closing eyes at rest modulates the functional connectivity of V1 with default and salience networks. Sci. Rep. 2020, 10, 1-10. [CrossRef]

61. Zhu, W.; Wen, W.; He, Y.; Xia, A.; Anstey, K.J.; Sachdev, P. Changing topological patterns in normal aging using large-scale structural networks. Neurobiol. Aging 2012, 33, 899-913. [CrossRef]

62. Wu, K.; Taki, Y.; Sato, K.; Qi, H.; Kawashima, R.; Fukuda, H. A longitudinal study of structural brain network changes with normal aging. Front. Hum. Neurosci. 2013, 7, 113. [CrossRef] 
63. Iordan, A.D.; Moored, K.D.; Katz, B.; Cooke, K.A.; Buschkuehl, M.; Jaeggi, S.M.; Polk, T.A.; Peltier, S.J.; Jonides, J.; Reuter-Lorenz, P.A. Age differences in functional network reconfiguration with working memory training. Hum. Brain Mapp. 2021, 42, 1888-1909. [CrossRef] [PubMed]

64. Chong, J.S.X.; Ng, K.K.; Tandi, J.; Wang, C.; Poh, J.-H.; Lo, J.C.; Chee, M.W.L.; Zhou, J.H. Longitudinal changes in the cerebral cortex functional organization of healthy elderly. J. Neurosci. 2019, 39, 5534-5550. [CrossRef] [PubMed]

65. La Corte, V.; Sperduti, M.; Malherbe, C.; Vialatte, F.; Lion, S.; Gallarda, T.; Oppenheim, C.; Piolino, P. Cognitive decline and reorganization of functional connectivity in healthy aging: The pivotal role of the salience network in the prediction of age and cognitive performances. Front. Aging Neurosci. 2016, 8, 204. [CrossRef] [PubMed]

66. Fjell, A.M.; Walhovd, K.B. Structural brain changes in aging: Courses, causes and cognitive consequences. Rev. Neurosci. 2010, 21, 187-222. [CrossRef] [PubMed]

67. Wang, L.; Wang, W.; Yan, T.; Song, J.; Yang, W.; Wang, B.; Go, R.; Huang, Q.; Wu, J. Beta-band functional connectivity influences audiovisual integration in older age: An EEG study. Front. Aging Neurosci. 2017, 9, 239. [CrossRef]

68. Cai, L.; Wei, X.; Wang, J.; Yu, H.; Deng, B.; Wang, R. Reconstruction of functional brain network in Alzheimer's disease via cross-frequency phase synchronization. Neurocomputing 2018, 314, 490-500. [CrossRef]

69. Wang, J.; Yang, C.; Wang, R.; Yu, H.; Cao, Y.; Liu, J. Functional brain networks in Alzheimer's disease: EEG analysis based on limited penetrable visibility graph and phase space method. Phys. A Stat. Mech. Its Appl. 2016, 460, 174-187. [CrossRef]

70. Vecchio, F.; Miraglia, F.; Maria Rossini, P. Connectome: Graph theory application in functional brain network architecture. Clin. Neurophysiol. Pract. 2017, 2, 206-213. [CrossRef]

71. Von Stein, A.; Sarnthein, J. Different frequencies for different scales of cortical integration: From local gamma to long range alpha/theta synchronization. Int. J. Psychophysiol. 2000, 38, 301-313. [CrossRef]

72. Guevara, M.A.; Paniagua, E.I.C.; González, M.H.; Carrillo, I.K.S.; Sepúlveda, M.L.A.; Orozco, J.C.H.; Gutiérrez, C.A. EEG activity during the spatial span task in young men: Differences between short-term and working memory. Brain Res. 2018, 1683, 86-94. [CrossRef]

73. Gola, M.; Magnuski, M.; Szumska, I.; Wróbel, A. EEG beta band activity is related to attention and attentional deficits in the visual performance of elderly subjects. Int. J. Psychophysiol. 2013, 89, 334-341. [CrossRef] [PubMed]

74. Teng, C.; Cheng, Y.; Wang, C.; Ren, Y.; Xu, W.; Xu, J. Aging-related changes of EEG synchronization during a visual working memory task. Cogn. Neurodyn. 2018, 12, 561-568. [CrossRef] [PubMed]

75. Moezzi, B.; Pratti, L.M.; Hordacre, B.; Graetz, L.; Berryman, C.; Lavrencic, L.M.; Ridding, M.C.; Keage, H.A.D.; McDonnell, M.D.; Goldsworthy, M.R. Characterization of young and old adult brains: An EEG functional connectivity analysis. Neuroscience 2019, 422, 230-239. [CrossRef] [PubMed]

76. Lotfan, S.; Shahyad, S.; Khosrowabadi, R.; Mohammadi, A.; Hatef, B. Support vector machine classification of brain states exposed to social stress test using EEG-based brain network measures. Biocybern. Biomed. Eng. 2019, 39, 199-213. [CrossRef] 